\title{
Properties of minimal charts and their applications VII: charts of type $(2,3,2)$
}

\author{
Teruo NAGASE and Akiko SHIMA 1
}

\begin{abstract}
Let $\Gamma$ be a chart, and we denote by $\Gamma_{m}$ the union of all the edges of label $m$. A chart $\Gamma$ is of type $(2,3,2)$ if there exists a label $m$ such that $w(\Gamma)=7, w\left(\Gamma_{m} \cap \Gamma_{m+1}\right)=2, w\left(\Gamma_{m+1} \cap \Gamma_{m+2}\right)=3$, and $w\left(\Gamma_{m+2} \cap \Gamma_{m+3}\right)=2$ where $w(G)$ is the number of white vertices in $G$. In this paper, we prove that there is no minimal chart of type $(2,3,2)$.
\end{abstract}

2010 Mathematics Subject Classification. Primary 57Q45; Secondary 57Q35.

Key Words and Phrases. surface link, chart, white vertex.

\section{Introduction}

Charts are oriented labeled graphs in a disk (see [1, [5], and see Section 2 for the precise definition of charts). From a chart, we can construct an oriented closed surface embedded in 4-space $\mathbb{R}^{4}$ (see [5, Chapter 14, Chapter 18 and Chapter 23]). A C-move is a local modification between two charts in a disk (see Section 2 for C-moves). A C-move between two charts induces an ambient isotopy between oriented closed surfaces corresponding to the two charts.

We will work in the PL category or smooth category. All submanifolds are assumed to be locally flat. In [16], we showed that there is no minimal chart with exactly five vertices (see Section 2 for the precise definition of minimal charts). Hasegawa proved that there exists a minimal chart with exactly six white vertices [2]. This chart represents a 2-twist spun trefoil. In [3] and [15, we investigated minimal charts with exactly four white vertices. In this paper, we investigate properties of minimal charts which are used to prove that there is no minimal chart with exactly seven white vertices (see [6], [7], [8], [9], [10], [11], [12]).

Let $\Gamma$ be a chart. For each label $m$, we denote by $\Gamma_{m}$ the union of all the edges of label $m$.

Now we define a type of a chart: Let $\Gamma$ be a chart with at least one white vertex, and $n_{1}, n_{2}, \ldots, n_{k}$ integers. The chart $\Gamma$ is of type $\left(n_{1}, n_{2}, \ldots, n_{k}\right)$ if there exists a label $m$ of $\Gamma$ satisfying the following three conditions:

(i) For each $i=1,2, \ldots, k$, the chart $\Gamma$ contains exactly $n_{i}$ white vertices in $\Gamma_{m+i-1} \cap \Gamma_{m+i}$.

(ii) If $i<0$ or $i>k$, then $\Gamma_{m+i}$ does not contain any white vertices.

\footnotetext{
${ }^{1}$ The second author is supported by JSPS KAKENHI Grant Number 18K03309.
} 
(iii) Both of the two subgraphs $\Gamma_{m}$ and $\Gamma_{m+k}$ contain at least one white vertex.

If we want to emphasize the label $m$, then we say that $\Gamma$ is of type $\left(m ; n_{1}, n_{2}, \ldots, n_{k}\right)$.

Note that $n_{1} \geq 1$ and $n_{k} \geq 1$ by the condition (iii).

We proved in [7, Theorem 1.1] that if there exists a minimal $n$-chart $\Gamma$ with exactly seven white vertices, then $\Gamma$ is a chart of type $(7),(5,2),(4,3),(3,2,2)$ or $(2,3,2)$ (if necessary we change the label $i$ by $n-i$ for all label $i$ ). In [10], we showed that there is no minimal chart of type $(3,2,2)$. In [11] and this paper, we shall show the following.

Theorem 1.1 There is no minimal chart of type $(2,3,2)$.

In the future paper [12], we shall show there is no minimal chart of type $(7),(5,2),(4,3)$. Therefore we shall show that there is no minimal chart with exactly seven white vertices.

The paper is organized as follows. In Section 2, we define charts and minimal charts. In Section 3, we investigate the graphs $\Gamma_{m+1}$ and $\Gamma_{m+2}$ for a minimal chart $\Gamma$ of type $(m ; 2,3,2)$. In Section 4, we consider a minimal chart $\Gamma$ of type $(m ; 2,3,2)$ such that $\Gamma_{m+1}$ contains either an oval (a graph with two white vertices and two black vertices, see Fig. 4(a)), or one of the two graphs as shown in Fig. 5 (the graphs with five white vertices). We investigate that the chart $\Gamma$ contains what kind of pseudo charts. In Section 5 , we give two examples of non minimal charts $\Gamma$ of type $(m ; 2,3,2)$ such that $\Gamma_{m+1}$ contains an oval. In Section 6, we review IO-Calculation (a property of numbers of inward arcs of label $k$ and outward arcs of label $k$ in a closed domain $F$ with $\partial F \subset \Gamma_{k-1} \cup \Gamma_{k} \cup \Gamma_{k+1}$ for some label $k$ ). In Section 7, we investigate a minimal chart $\Gamma$ of type $(m ; 2,3,2)$ such that $\Gamma_{m+1}$ contains an oval. We shall show that the chart $\Gamma$ contains the pseudo chart as shown in Fig. 17(a) by C-moves without changing types of charts. In Section 8, we shall show that neither $\Gamma_{m+1}$ nor $\Gamma_{m+2}$ contains an oval for any minimal chart $\Gamma$ of type $(m ; 2,3,2)$. In Section 9 , we investigate a minimal chart $\Gamma$ of type $(m ; 2,3,2)$ such that $\Gamma_{m+1}$ contains the graph as shown in Fig. 5(a). In Section 10, we shall show that neither $\Gamma_{m+1}$ nor $\Gamma_{m+2}$ contains the graph as shown in Fig. 5(a) for any minimal chart $\Gamma$ of type $(m ; 2,3,2)$. In Section 11 , we give an example of a non minimal chart $\Gamma$ of type $(m ; 2,3,2)$ such that $\Gamma_{m+1}$ contains the graph as shown in Fig. 5(b). In Section 12 , we shall prove Theorem 1.1.

\section{Preliminaries}

In this section, we introduce the definition of charts and its related words.

Let $n$ be a positive integer. An $n$-chart (a braid chart of degree $n$ [1] or a surface braid chart of degree $n[5]$ ) is an oriented labeled graph in the interior of a disk, which may be empty or have closed edges without vertices satisfying the following four conditions (see Fig. 11): 
(i) Every vertex has degree 1, 4, or 6 .

(ii) The labels of edges are in $\{1,2, \ldots, n-1\}$.

(iii) In a small neighborhood of each vertex of degree 6 , there are six short arcs, three consecutive arcs are oriented inward and the other three are outward, and these six are labeled $i$ and $i+1$ alternately for some $i$, where the orientation and label of each arc are inherited from the edge containing the arc.

(iv) For each vertex of degree 4, diagonal edges have the same label and are oriented coherently, and the labels $i$ and $j$ of the diagonals satisfy $|i-j|>1$.

We call a vertex of degree 1 a black vertex, a vertex of degree 4 a crossing, and a vertex of degree 6 a white vertex respectively.

Among six short arcs in a small neighborhood of a white vertex, a central arc of each three consecutive arcs oriented inward (resp. outward) is called a middle arc at the white vertex (see Fig. 1(c)). For each white vertex $v$, there are two middle arcs at $v$ in a small neighborhood of $v$. An edge is said to be middle at a white vertex $v$ if it contains a middle arc at $v$.

Let $e$ be an edge connecting $v_{1}$ and $v_{2}$. If $e$ is oriented from $v_{1}$ to $v_{2}$, then we say that $e$ is oriented outward at $v_{1}$ and inward at $v_{2}$

(a)

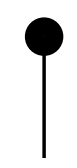

(b)

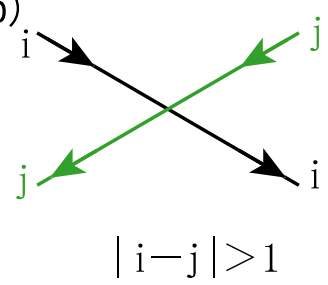

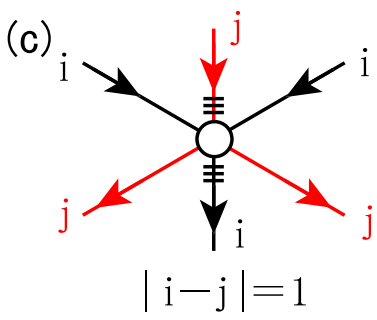

Figure 1: (a) A black vertex. (b) A crossing. (c) A white vertex. Each arc with three transversal short arcs is a middle arc at the white vertex.

Now $C$-moves are local modifications of charts as shown in Fig. 2] (cf. [1], [5] and [17]). Two charts are said to be $C$-move equivalent if there exists a finite sequence of C-moves which modifies one of the two charts to the other.

An edge in a chart is called a free edge if it has two black vertices.

For each chart $\Gamma$, let $w(\Gamma)$ and $f(\Gamma)$ be the number of white vertices, and the number of free edges respectively. The pair $(w(\Gamma),-f(\Gamma))$ is called a complexity of the chart (see [4]). A chart $\Gamma$ is called a minimal chart if its complexity is minimal among the charts $\mathrm{C}$-move equivalent to the chart $\Gamma$ with respect to the lexicographic order of pairs of integers.

We showed the difference of a chart in a disk and in a 2-sphere (see [6, Lemma 2.1]). This lemma follows from that there exists a natural one-toone correspondence between $\left\{\right.$ charts in $\left.S^{2}\right\} / \mathrm{C}$-moves and $\left\{\right.$ charts in $\left.D^{2}\right\} / \mathrm{C}$ - 

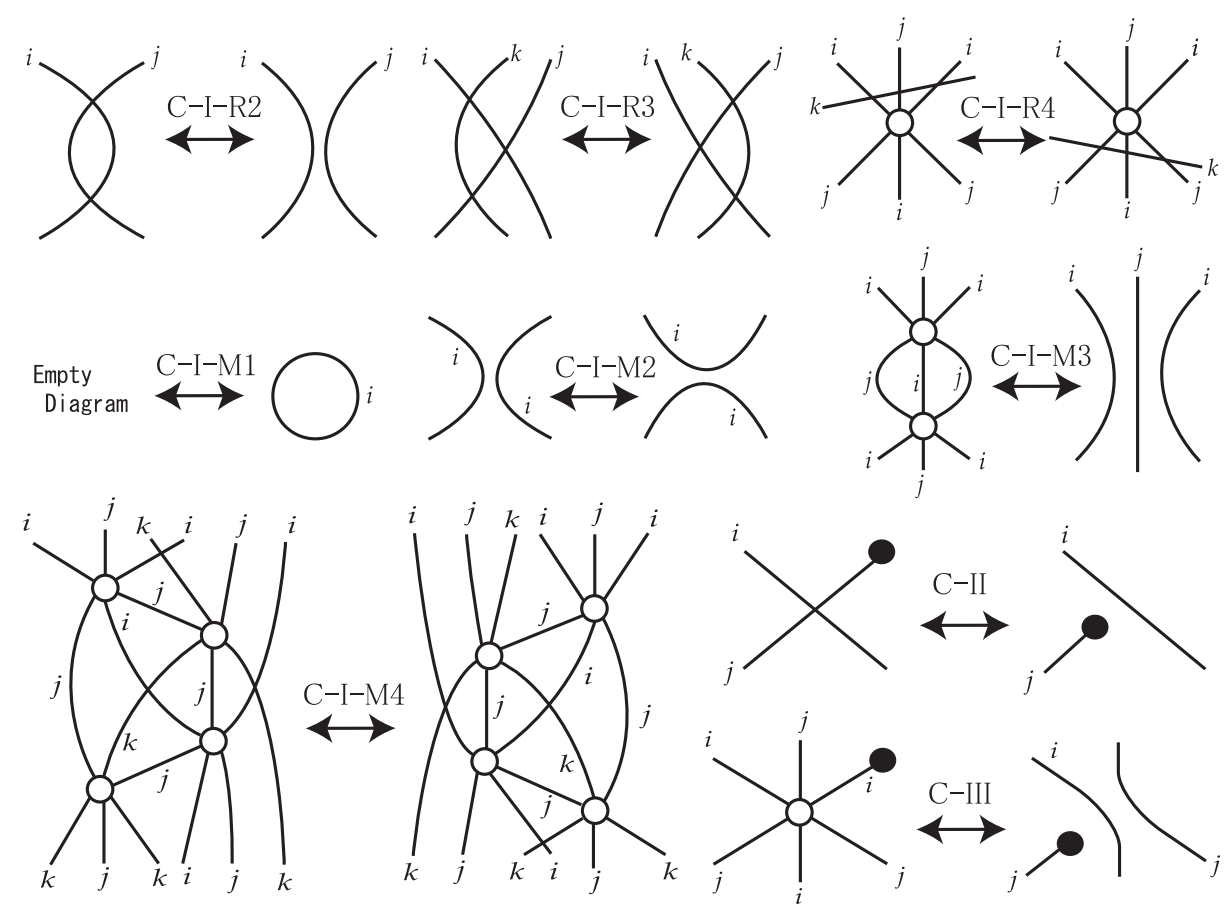

Figure 2: For the C-III move, the edge with the black vertex is not middle at a white vertex in the left figure.

moves, conjugations ([5, Chapter 23 and Chapter 25]). To make the argument simple, we assume that the charts lie on the 2-sphere instead of the disk.

Assumption 1 In this paper, all charts are contained in the 2-sphere $S^{2}$.

We have the special point in the 2 -sphere $S^{2}$, called the point at infinity, denoted by $\infty$. In this paper, all charts are contained in a disk such that the disk does not contain the point at infinity $\infty$.

An edge in a chart is called a terminal edge if it has a white vertex and a black vertex.

Let $\Gamma$ be a chart, and $m$ a label of $\Gamma$. A hoop is a closed edge of $\Gamma$ without vertices (hence without crossings, neither). A ring is a simple closed curve in $\Gamma_{m}$ containing a crossing but not containing any white vertices. A hoop is said to be simple if one of the two complementary domains of the hoop does not contain any white vertices.

We can assume that all minimal charts $\Gamma$ satisfy the following four conditions (see [6], [7], [8], [14]):

Assumption 2 If an edge of $\Gamma$ contains a black vertex, then the edge is a free edge or a terminal edge. Moreover any terminal edge contains a middle arc.

Assumption 3 All free edges and simple hoops in $\Gamma$ are moved into a small neighborhood $U_{\infty}$ of the point at infinity $\infty$. Hence we assume that $\Gamma$ does not contain free edges nor simple hoops, otherwise mentioned. 
Assumption 4 Each complementary domain of any ring and hoop must contain at least one white vertex.

Assumption 5 The point at infinity $\infty$ is moved in any complementary domain of $\Gamma$.

In this paper for a set $X$ in a space we denote the interior of $X$, the boundary of $X$ and the closure of $X$ by $\operatorname{Int} X, \partial X$ and $C l(X)$ respectively.

Let $\alpha$ be a simple arc or an edge, and $p, q$ the endpoints of $\alpha$. We denote $\partial \alpha=\{p, q\}$ and Int $\alpha=\alpha-\partial \alpha$.

\section{The graphs $\Gamma_{m+1}$ and $\Gamma_{m+2}$}

In this section, we investigate the graphs $\Gamma_{m+1}$ and $\Gamma_{m+2}$ for a minimal chart $\Gamma$ of type $(m ; 2,3,2)$.

In our argument we often construct a chart $\Gamma$. On the construction of a chart $\Gamma$, for a white vertex $w \in \Gamma_{m}$ for some label $m$, among the three edges of $\Gamma_{m}$ containing $w$, if one of the three edges is a terminal edge (see Fig. 3(a) and (b)), then we remove the terminal edge and put a black dot at the center of the white vertex as shown in Fig. 3(c). Namely Fig. 3(c) means Fig. 3(a) or Fig. 3(b). We call the vertex in Fig. 3(c) a $B W$-vertex with respect to $\Gamma_{m}$.



(d) (b)



(e)

(c)

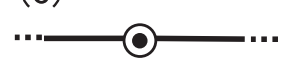

Figure 3: (a),(b) White vertices in terminal edges. (c),(d),(e) BW-vertices.

Lemma 3.1 ([10, Lemma 3.1]) In a minimal chart $\Gamma$, for each $B W$-vertex in $\Gamma_{m}$, the two edges of label $m$ containing the $B W$-vertex are oriented inward or outward at the $B W$-vertex simultaneously if each of the two edges is not a terminal edge (see Fig. $3(d)$ and $(e)$ ).

Let $X$ be a set in a chart $\Gamma$. Let

$$
w(X)=\text { the number of white vertices in } X \text {. }
$$

Lemma 3.2 ([10, Lemma 3.2(1)]) Let $\Gamma$ be a minimal chart, and $m$ a label of $\Gamma$. Let $G$ be a connected component of $\Gamma_{m}$. If $1 \leq w(G)$, then $2 \leq w(G)$.

We call an oval, a skew $\theta$-curve the two graphs as shown in Fig. 4(a),(b) respectively.

Lemma 3.3 ([11, Theorem 1.3]) If there exists a minimal chart $\Gamma$ of type $(m ; 2,3,2)$, then each of $\Gamma_{m+1}$ and $\Gamma_{m+2}$ contains either the union of an oval and a skew $\theta$-curve, or one of two graphs as shown in Fig. 5. 

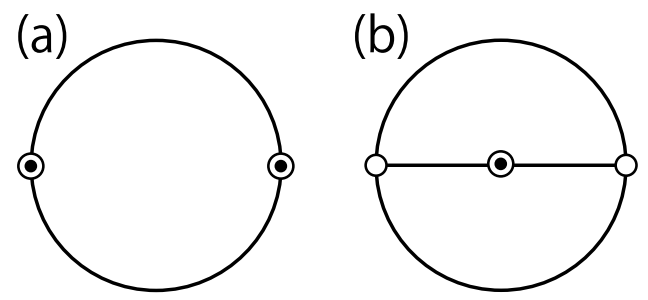

Figure 4: (a) An oval. (b) A skew $\theta$-curve.

(a)

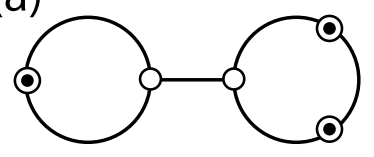

(b)

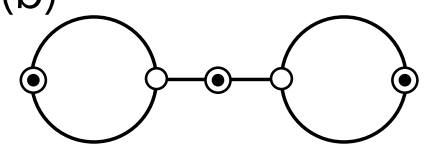

Figure 5: Graphs with three black vertices.

\section{RO-families of pseudo charts}

In this section we consider a minimal chart $\Gamma$ of type $(m ; 2,3,2)$ such that $\Gamma_{m+1}$ contains either an oval, or one of the two graphs as shown in Fig. 5. We investigate that the chart $\Gamma$ contains what kind of pseudo charts.

Let $\Gamma$ be a chart. Suppose that an object consists of some edges of $\Gamma$, arcs in edges of $\Gamma$ and arcs around white vertices. Then the object is called a pseudo chart.

Let $\Gamma$ be a chart, $D$ a disk, and $G$ a pseudo chart with $G \subset D$. Let $r: D \rightarrow$ $D$ be a reflection of $D$, and $G^{*}$ the pseudo chart obtained from $G$ by changing the orientations of all of the edges. Then the set $\left\{G, G^{*}, r(G), r\left(G^{*}\right)\right\}$ is called the RO-family of the pseudo chart $G$.

In our argument, we often need a name for an unnamed edge by using a given edge and a given white vertex. For the convenience, we use the following naming: Let $e^{\prime}, e_{i}, e^{\prime \prime}$ be three consecutive edges containing a white vertex $w_{j}$. Here, the two edges $e^{\prime}$ and $e^{\prime \prime}$ are unnamed edges. There are six $\operatorname{arcs}$ in a neighborhood $U$ of the white vertex $w_{j}$. If the three $\operatorname{arcs} e^{\prime} \cap U$, $e_{i} \cap U, e^{\prime \prime} \cap U$ lie anticlockwise around the white vertex $w_{j}$ in this order, then $e^{\prime}$ and $e^{\prime \prime}$ are denoted by $a_{i j}$ and $b_{i j}$ respectively (see Fig. 6). There is a possibility $a_{i j}=b_{i j}$ if they contain only one white vertex.

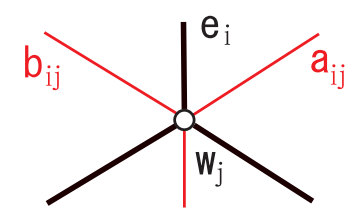

Figure 6: The three edges $a_{i j}, e_{i}, b_{i j}$ are consecutive edges around the white vertex $w_{j}$.

Lemma 4.1 ([11, Lemma 8.3]) Let $\Gamma$ be a minimal chart of type $(m ; 2,3,2)$. 

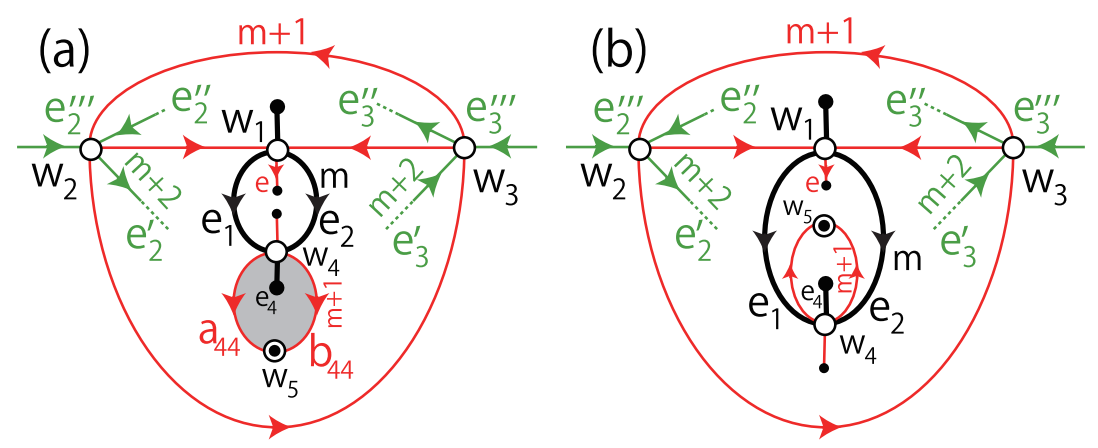

Figure 7: Pseudo charts containing a skew $\theta$-curve and an oval of label $m+1$.


Figure 8: (a),(b) Pseudo charts containing the graph as shown in Fig. 5(a). (c),(d) Pseudo charts containing the graph as shown in Fig. 5(b).

If $\Gamma_{m+1}$ contain an oval, then $\Gamma$ contains one of the $R O$-families of the two pseudo charts as shown in Fig. 7 .

Lemma 4.2 ([11, Lemma 8.4]) Let $\Gamma$ be a minimal chart of type $(m ; 2,3,2)$. If $\Gamma_{m+1}$ contains the graph as shown in Fig. $5(a)$, then $\Gamma$ contains one of the RO-families of the two pseudo charts as shown in Fig. $8(a),(b)$.

Lemma 4.3 ([11, Lemma 8.5]) Let $\Gamma$ be a minimal chart of type $(m ; 2,3,2)$. If $\Gamma_{m+1}$ contains the graph as shown in Fig. $5(b)$, then $\Gamma$ contains one of the RO-families of the two pseudo charts as shown in Fig. $8(c),(d)$.

Lemma 4.4 Let $\Gamma$ be a minimal chart of type $(m ; 2,3,2)$. If $\Gamma_{m+2}$ contains one of the two graphs as shown in Fig. 5, then $\Gamma$ contains one of the ROfamilies of the four pseudo charts as shown in Fig. 9. 



Figure 9: (a),(b) Pseudo charts containing the graph as shown in Fig. 5(a). (c),(d) Pseudo charts containing the graph as shown in Fig. 5(b).

Proof. Let $\Gamma$ be a minimal chart of type $(m ; 2,3,2)$ such that $\Gamma_{m+2}$ contains the graph as shown in Fig. 5(a). Let $\Gamma^{\prime}$ be the chart obtained from $\Gamma$ by changing labels $\cdots, m, m+1, m+2, m+3, \cdots$ into $\cdots, m+3, m+2, m+$ $1, m, \cdots$, respectively. Then $\Gamma^{\prime}$ is a minimal chart of type $(m ; 2,3,2)$ such that $\Gamma_{m+1}^{\prime}$ contains the graph as shown in Fig. 5(a). Thus by Lemma 4.2, the chart $\Gamma^{\prime}$ contains one of the RO-families of the two pseudo charts as shown in Fig. 8(a),(b). Hence $\Gamma$ contains one of the RO-families of the two pseudo charts as shown in Fig. 9(a),(b).

Similarly we can show this lemma for the case that $\Gamma_{m+2}$ contains the graph as shown in Fig. $5(\mathrm{~b})$.

\section{Rings}

In this section, we give two examples of non minimal charts $\Gamma$ of type $(m ; 2,3,2)$ such that $\Gamma_{m+1}$ contains an oval.

Lemma 5.1 (8, Lemma 6.1]) Let $\Gamma$ be a minimal chart. Let $C$ be a ring or a non simple hoop, and $D$ a disk with $\partial D=C$. If $w(\Gamma \cap D)=1$, then $\Gamma$ is $C$-move equivalent to the minimal chart $C l(\Gamma-C)$.

Let $\Gamma$ be a chart, $m$ a label of $\Gamma, D$ a disk with $\partial D \subset \Gamma_{m}$, and $k$ a positive integer. If $\partial D$ contains exactly $k$ white vertices, then $D$ is called $a k$-angled disk of $\Gamma_{m}$. Note that the boundary $\partial D$ may contain crossings.

Let $\Gamma$ be a chart, and $m$ a label of $\Gamma$. An edge of label $m$ is called a feeler of a $k$-angled disk $D$ of $\Gamma_{m}$ if the edge intersects $N-\partial D$ where $N$ is a regular neighborhood of $\partial D$ in $D$. 


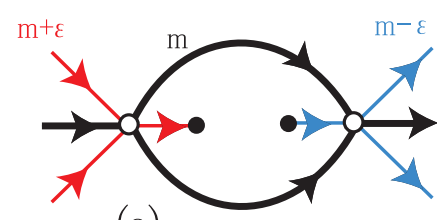

(a)

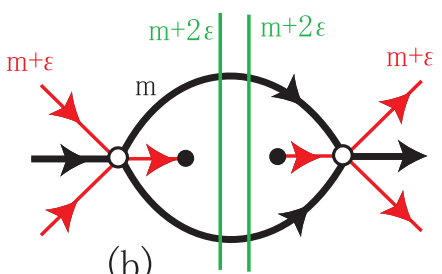

(b)

Figure 10: $m$ is a label, and $\varepsilon \in\{+1,-1\}$.

Lemma 5.2 ([7, Corollary 5.8]) Let $\Gamma$ be a minimal chart. Let $D$ be a 2angled disk of $\Gamma_{m}$ with at most one feeler. If $w(\Gamma \cap \operatorname{Int} D)=0$, then a regular neighborhood of D contains one of two pseudo charts as shown in Fig. 10.

Let $\Gamma$ be a chart, and $m$ a label of $\Gamma$. Let $L$ be the closure of a connected component of the set obtained by taking out all the white vertices from $\Gamma_{m}$. If $L$ contains at least one white vertex but does not contain any black vertex, then $L$ is called an internal edge of label $m$. Note that an internal edge may contain a crossing of $\Gamma$.

A simple arc $\alpha$ in a compact surface $F$ is said to be proper if $\alpha \cap \partial F=\partial \alpha$.

Lemma 5.3 Let $\Gamma$ be a chart of type $(m ; 2,3,2)$. If $\Gamma$ contains one of the two pseudo charts as shown in Fig. $11(a),(b)$, then $\Gamma$ is not minimal.
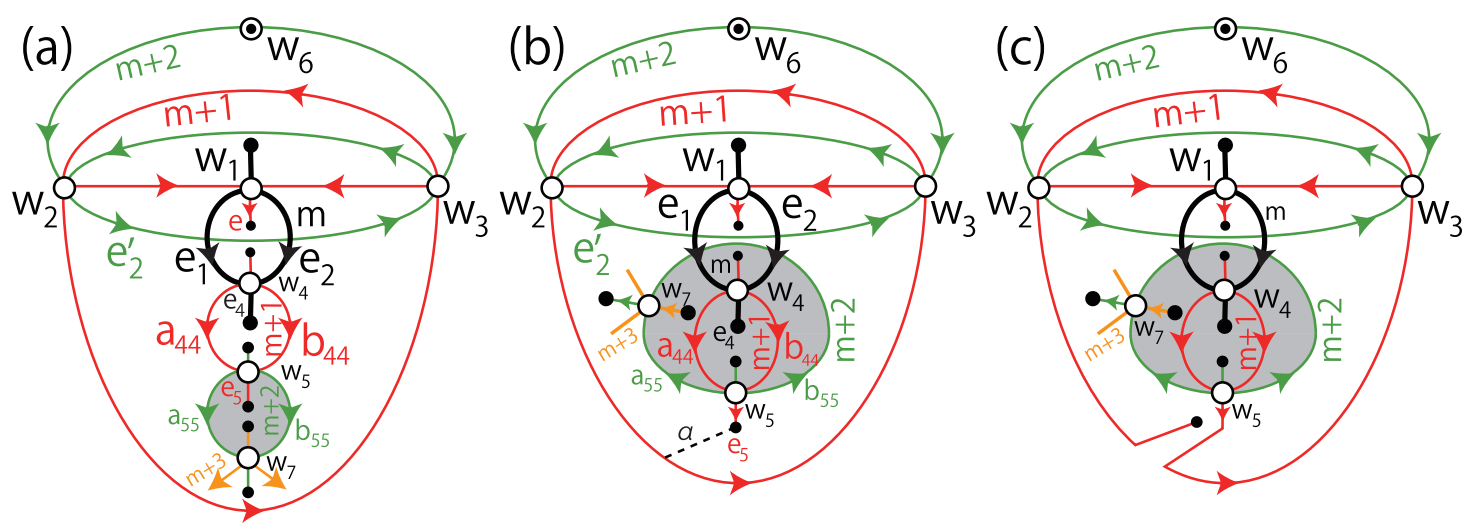

Figure 11: (a) The gray region $F_{2}$ does not contain $w_{4}$. (b) The gray region $F_{2}$ contains $w_{4}$. (c) The terminal edge of label $m+1$ at $w_{2}$ is not middle at $w_{2}$.

Proof. Suppose that $\Gamma$ is minimal. We use the notations as shown in Fig. 11(a),(b), where $a_{44}, b_{44}$ are internal edges of label $m+1$ at $w_{4}, e_{2}^{\prime}$ is the internal edge of label $m+2$ connecting $w_{2}$ and $w_{3}$, and $a_{55}, b_{55}$ are internal edges of label $m+2$ at $w_{5}$.

Since $w_{1}, w_{2}, w_{3}, w_{4}, w_{5} \in \Gamma_{m+1}, w_{6}, w_{7} \in \Gamma_{m+2}$ and $\Gamma$ is of type $(m ; 2,3,2)$, we have

(1) $\Gamma_{m+3}$ contains exactly two white vertices $w_{6}, w_{7}$. 
Let $D_{1}$ be the 3 -angled disk of $\Gamma_{m+1}$ with $\partial D_{1} \ni w_{1}, w_{2}, w_{3}$ and $D_{1} \ni w_{4}$.

Claim 1. There is no ring of label $m+2$ in $D_{1}$.

Proof of Claim 1. Suppose that there exists a ring $R$ of label $m+2$ in $D_{1}$. Then the ring $R$ does not intersect the two internal edges $a_{44}, b_{44}$ of label $m+1$ and the three internal edges $e_{2}^{\prime}, a_{55}, b_{55}$ of label $m+2$. Thus the ring $R$ is contained in a connected component of $\operatorname{Int} D_{1}-\left(a_{44} \cup b_{44} \cup e_{2}^{\prime} \cup a_{55} \cup b_{55}\right)$, say $F$. Since each connected component of Int $D_{1}-\left(a_{44} \cup b_{44} \cup e_{2}^{\prime} \cup a_{55} \cup b_{55}\right)$ does not contain any white vertex, the ring $R$ does not bound a disk in $F$ by Assumption 4. Hence $F$ is the open annulus with $\partial F \supset e_{2}^{\prime} \cup a_{55} \cup b_{55}$.

Since $w_{7} \in \partial F$ and $w_{6} \notin D_{1}$, the two white vertices $w_{6}, w_{7}$ of $\Gamma_{m+3}$ is separated by the ring $R$ of label $m+2$. Thus by (1), there exists a connected component of $\Gamma_{m+3}$ with only one white vertex. This contradicts Lemma 3.2 . Hence there is no ring of label $m+2$ in $D_{1}$. Hence Claim 1 holds.

Claim 2. By C-moves without changing type of charts, we can assume that there is no ring of label $m, m+1$ in $D_{1}$.

Proof of Claim 2. Suppose that there exists a ring $R$ of label $m$ or $m+1$ in $D_{1}$. Then by the similar way of the proof of Claim 1 , the ring $R$ bounds a disk containing only one white vertex $w_{7}$. Hence by Lemma 5.1, the chart $\Gamma$ is C-move equivalent to $C l(\Gamma-R)$. Thus we can assume that there is no ring of label $m, m+1$ in $D_{1}$. Hence Claim 2 holds.

Now we are ready to prove Lemma 5.3. Suppose that $\Gamma$ contains the pseudo chart as shown in Fig. 11(a). Let $D$ be the 2-angled disk of $\Gamma_{m}$ in $D_{1}$ with $\partial D \ni w_{1}, w_{2}$. Then $e_{2}^{\prime} \cap D$ is a proper arc of $D$ of label $m+2$. By Lemma 5.2, a regular neighborhood of $D$ contains the pseudo chart as shown in Fig. 10(b). Thus there exists another proper arc of $D$ contained in a ring of label $m+2$. This contradicts Claim 1 .

Suppose that $\Gamma$ contains the pseudo chart as shown in Fig. 11(b). Let $e_{5}$ be the terminal edge of label $m+1$ at $w_{5}$. Let $\alpha$ be an arc in $D_{1}$ connecting the black vertex of $e_{5}$ and a point in $\partial D_{1}$ (see Fig. 11(b)). By Claim 1 and Claim 2, we can assume that $\left(\Gamma_{m} \cup \Gamma_{m+1} \cap \Gamma_{m+2}\right) \cap \operatorname{Int} \alpha=\emptyset$. Thus we can apply C-II moves and a C-I-M2 move between the edge $e_{5}$ and $\partial D_{1}$ along the arc $\alpha$ so that we obtain a new terminal edge of label $m+1$ at $w_{2}$ not middle at $w_{2}$ (see Fig. 11(c)). This contradicts Assumption 2.

Therefore $\Gamma$ is not minimal. We complete the proof of Lemma 5.3 .

\section{IO-Calculation}

In this section, we review a useful technic, called IO-Calculation.

Let $\Gamma$ be a chart, and $v$ a vertex. Let $\alpha$ be a short arc of $\Gamma$ in a small neighborhood of $v$ with $v \in \partial \alpha$. If the arc $\alpha$ is oriented to $v$, then $\alpha$ is called an inward arc, and otherwise $\alpha$ is called an outward arc. 
Let $\Gamma$ be an $n$-chart. Let $F$ be a closed domain with $\partial F \subset \Gamma_{k-1} \cup \Gamma_{k} \cup \Gamma_{k+1}$ for some label $k$ of $\Gamma$, where $\Gamma_{0}=\emptyset$ and $\Gamma_{n}=\emptyset$. By Condition (iii) for charts, in a small neighborhood of each white vertex, there are three inward arcs and three outward arcs. Also in a small neighborhood of each black vertex, there exists only one inward arc or one outward arc. We often use the following fact, when we fix (inward or outward) arcs near white vertices and black vertices:

(*) The number of inward arcs contained in $F \cap \Gamma_{k}$ is equal to the number of outward arcs in $F \cap \Gamma_{k}$.

When we use this fact, we say that we use IO-Calculation with respect to $\Gamma_{k}$ in $F$. For example, in a minimal chart $\Gamma$, consider the pseudo chart as shown in Fig. 12 where

(1) $F$ is an annulus with $\partial F \subset \Gamma_{k-1}$,

(2) $v_{1}, v_{2}, v_{3}, v_{4}, v_{5}$ are white vertices in $\partial F$ with $v_{1}, v_{4} \in \Gamma_{k-2} \cap \Gamma_{k-1}$ and $v_{2}, v_{3}, v_{5} \in \Gamma_{k-1} \cap \Gamma_{k}$,

(3) $e_{2}$ is an edge of label $k$ oriented outward at $v_{2}$,

(4) $e_{3}$ is an edge of label $k$ oriented inward at $v_{3}$ but not middle at $v_{3}$,

(5) $a_{55}, b_{55}$ are edges of label $k$ oriented outward at $v_{5}$ but not middle at $v_{5}$.

Then we can show that $w(\Gamma \cap \operatorname{Int} F) \geq 1$. Suppose $w(\Gamma \cap \operatorname{Int} F)=0$. By (4) and (5), none of $e_{3}, a_{55}, b_{55}$ are middle at $v_{3}$ or $v_{5}$. Thus by Assumption 2 ,

(6) none of $e_{3}, a_{55}, b_{55}$ are terminal edges.

If $e_{2}$ is a terminal edge, then the number of inward arcs in $F \cap \Gamma_{k}$ is two, but the number of outward arcs in $F \cap \Gamma_{k}$ is three. This contradicts the fact $(*)$. If $e_{2}$ is not a terminal edge, then we have the same contradiction. Thus $w(\Gamma \cap \operatorname{Int} F) \geq 1$. Instead of the above argument, we just say that

we have $w(\Gamma \cap \operatorname{Int} F) \geq 1$ by IO-Calculation with respect to $\Gamma_{k}$ in $F$.

Lemma 6.1 (Triangle Lemma) ([9, Lemma 8.3(2)]) For a minimal chart $\Gamma$, if there exists a 3-angled disk $D_{1}$ of $\Gamma_{m}$ without feelers in a disk $D$ as shown in Fig. $13(a)$, then $w\left(\Gamma \cap \operatorname{Int} D_{1}\right) \geq 1$.

Corollary 6.2 (Corollary of Triangle Lemma) Let $\Gamma$ be a minimal chart. Let $D_{1}$ be a 3-angled disk of $\Gamma_{m}$ with at most one feeler in a disk $D$ as shown in Fig. $13(b)$. Let $w_{1}, w_{2}, w_{3}$ be the white vertices in $\partial D_{1}$ with $w_{1}, w_{2} \in \Gamma_{m+\varepsilon}$ $(\varepsilon \in\{+1,-1\})$. If $w_{3} \in \Gamma_{m-\varepsilon}$, then $w\left(\Gamma \cap \operatorname{Int} D_{1}\right) \geq 1$. 


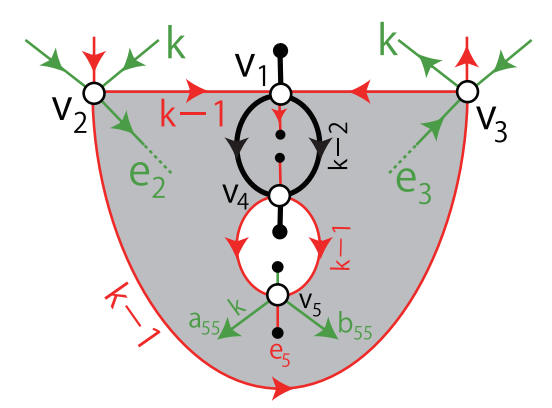

Figure 12: The gray region is the annulus $F$.
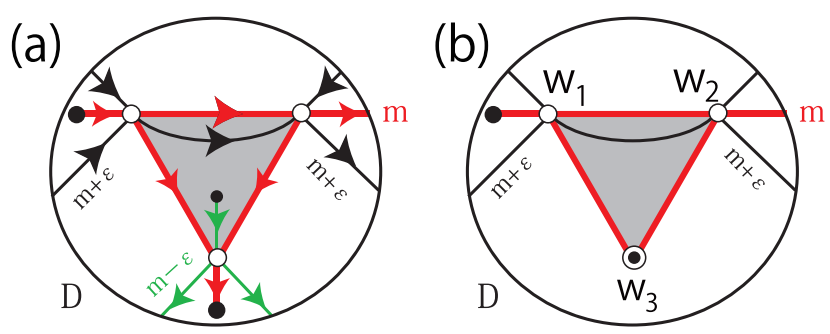

Figure 13: The gray region is the 3 -angled disk $D_{1}$. The thick lines are edges of label $m$, and $\varepsilon \in\{+1,-1\}$.

Proof. Let $e$ be the terminal edge of label $m$ at $w_{3}$. Without loss of generality we can assume that the edge $e$ is oriented outward at $w_{3}$.

If $e \not \subset D_{1}$, then the 3 -angled disk $D_{1}$ is as shown in Fig. 13(a). Thus by Lemma 6.1, we have $w\left(\Gamma \cap \operatorname{Int} D_{1}\right) \geq 1$.

If $e \subset D_{1}$, then there exist two internal edges $e^{\prime}, e^{\prime \prime}$ (possibly terminal edges) of label $m-\varepsilon$ at $w_{3}$ in $D_{1}$. By Assumption 2, neither $e^{\prime}$ nor $e^{\prime \prime}$ is a terminal edge, and both of $e^{\prime}, e^{\prime \prime}$ are oriented outward at $w_{3}$. Thus we have $w\left(\Gamma \cap \operatorname{Int} D_{1}\right) \geq 1$ by IO-Calculation with respect to $\Gamma_{m-\varepsilon}$ in $D_{1}$.

\section{$7 \quad \mathrm{X}$-change Lemma}

In this section we investigate a minimal chart $\Gamma$ of type $(m ; 2,3,2)$ such that $\Gamma_{m+1}$ contains an oval. We shall show that the chart $\Gamma$ contains the pseudo chart as shown in Fig. 17(a) by C-moves without changing types of charts.

Let $\Gamma$ and $\Gamma^{\prime}$ be C-move equivalent charts. Suppose that a pseudo chart $X$ of $\Gamma$ is also a pseudo chart of $\Gamma^{\prime}$. Then we say that $\Gamma$ is modified to $\Gamma^{\prime}$ by $C$-moves keeping $X$ fixed. In Fig. 14, we give examples of C-moves keeping pseudo charts fixed.

Let $\Gamma$ be a chart and $m$ a label. An oval $G$ of $\Gamma_{m+1}$ is said to be special, if there exists a 2-angled disk $D$ of $\Gamma_{m+1}$ without feelers such that $\partial D \subset G$, $w(\Gamma \cap \operatorname{Int} D)=0$, the disk $D$ contains a terminal edge of label $m$ and a terminal edge of label $m+2$, but $D$ does not contain any free edges, hoops nor crossings (see Fig. 15(a)). 

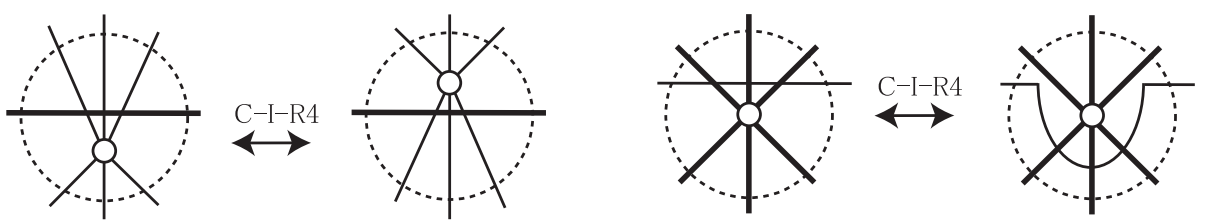

Figure 14: C-moves keeping thicken figures fixed.

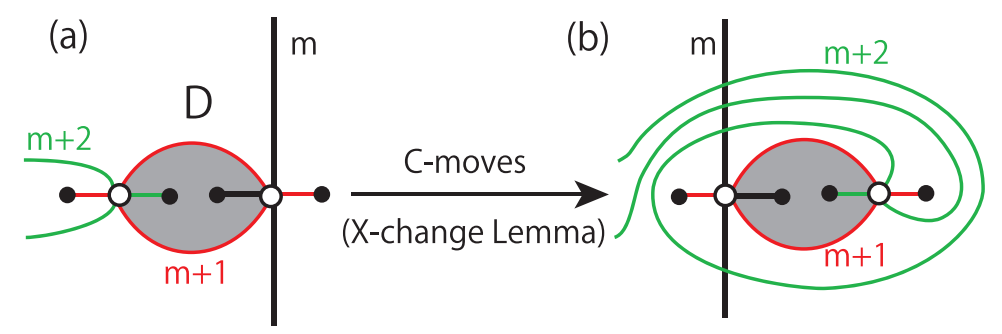

Figure 15: The gray regions are the disk $D$.

Lemma 7.1 ([3, Lemma 6.1 and Lemma 6.3]) Let $\Gamma$ be a chart. Let $G$ be an oval of $\Gamma_{m+1}$ and $D$ a 2-angled disk of $\Gamma_{m+1}$ without feelers such that $\partial D \subset G$ and $w(\Gamma \cap \operatorname{Int} D)=0$.

(a) (X-change Lemma) If $G$ is a special oval in a minimal chart $\Gamma$, then the chart $\Gamma$ is $C$-move equivalent to the chart obtained from $\Gamma$ by replacing a regular neighborhood of $D$ with the pseudo chart as shown in Fig. 15.(b).

(b) If $D$ contains a terminal edge of label $m$ and a terminal edge of label $m+2$, then $G$ can be modified to a special oval by $C$-moves in a regular neighborhood of $D$ keeping $G \cup \Gamma_{m} \cup \Gamma_{m+2}$ fixed.

Lemma 7.2 ([10, Lemma 4.4(2)]) Let $\Gamma$ be a minimal chart, and $m$ a label of $\Gamma$. Let $D$ be a 2 -angled disk of $\Gamma_{m}$ with exactly one feeler. If $w(\Gamma \cap \operatorname{Int} D)=1$, then a regular neighborhood of $D$ contains one of the $R O$-family of the pseudo chart as shown in Fig. 16.



Figure 16: $m$ is a label, $\varepsilon, \delta \in\{+1,-1\}$.

Lemma 7.3 Let $\Gamma$ be a minimal chart of type $(m ; 2,3,2)$. If $\Gamma$ contains the pseudo chart as shown in Fig. $7($ a), then the chart $\Gamma$ is $C$-move equivalent to 


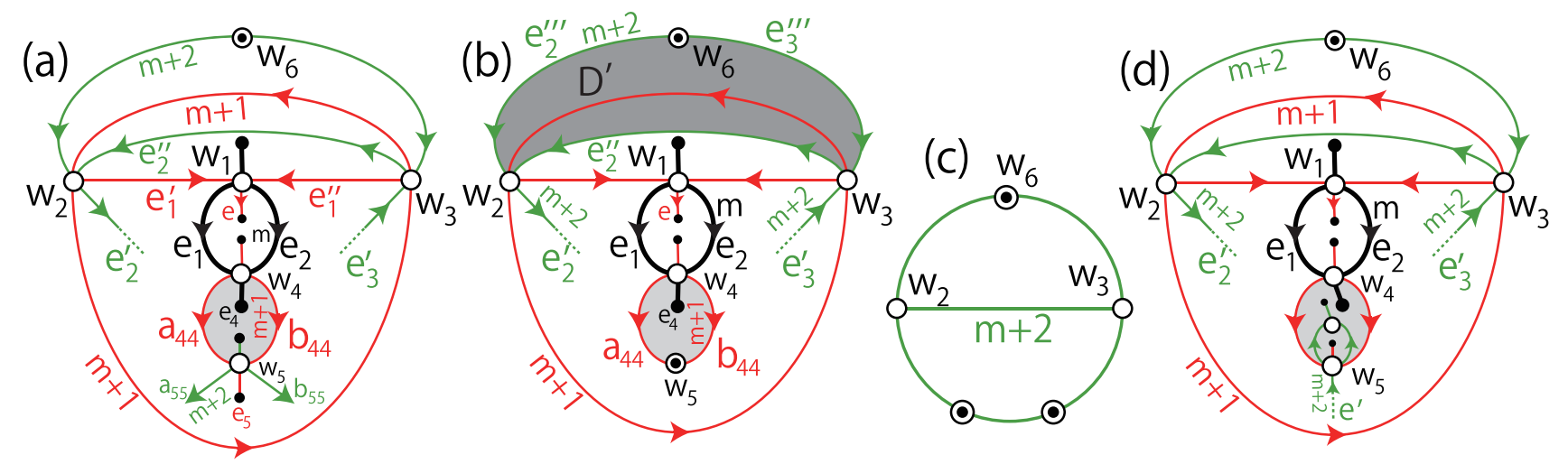

Figure 17: The light gray regions are the disk $E$. The dark gray region is the disk $D^{\prime}$.

a minimal chart of type $(m ; 2,3,2)$ containing the pseudo chart as shown in Fig. 17 (a). Moreover $e_{2}^{\prime}=e_{3}^{\prime}$ and $a_{55} \cap b_{55}$ contains a white vertex different from $w_{5}$, where $e_{2}^{\prime}, e_{3}^{\prime}, a_{55}, b_{55}$ are internal edges of label $m+2$ at $w_{2}, w_{3}, w_{5}, w_{5}$ respectively.

Proof. We use the notations as shown in Fig. 7(a). Now $\Gamma_{m+1}$ contains a skew $\theta$-curve (see Fig. 7(a)). The skew $\theta$-curve separates $S^{2}$ into three disks. Let $D_{1}, D_{2}, D_{3}$ be the three disks such that $D_{1} \supset a_{44} \cup b_{44}$, the disk $D_{2}$ contains the terminal edge of label $m$ at $w_{1}$, and $D_{3}$ is a 2-angled disk.

Let $e_{2}^{\prime}, e_{2}^{\prime \prime}, e_{2}^{\prime \prime \prime}$ be internal edges (possibly terminal edges) of label $m+2$ at $w_{2}$ in $D_{1}, D_{2}, D_{3}$ respectively. Let $e_{3}^{\prime}, e_{3}^{\prime \prime}, e_{3}^{\prime \prime \prime}$ be internal edges (possibly terminal edges) of label $m+2$ at $w_{3}$ in $D_{1}, D_{2}, D_{3}$ respectively (see Fig. 7 (a)). Then

(1) the two edges $e_{2}^{\prime \prime \prime}, e_{3}^{\prime \prime \prime}$ are oriented inward at $w_{2}, w_{3}$ respectively,

(2) the edge $e_{2}^{\prime}$ is oriented outward at $w_{2}$.

Claim 1. $w\left(\Gamma \cap \operatorname{Int} D_{3}\right) \geq 1$.

Proof of Claim 1. We can show that neither $e_{2}^{\prime \prime \prime}$ nor $e_{3}^{\prime \prime \prime}$ is middle at $w_{2}$ or $w_{3}$. Thus by Assumption 2 ,

(3) neither $e_{2}^{\prime \prime \prime}$ nor $e_{3}^{\prime \prime \prime}$ is a terminal edge.

Hence (1) implies that $w\left(\Gamma \cap \operatorname{Int} D_{3}\right) \geq 1$ by IO-Calculation with respect to $\Gamma_{m+2}$ in $D_{3}$. Thus Claim 1 holds.

We shall show $w_{5} \in \Gamma_{m+1} \cap \Gamma_{m+2}$. Since $w_{1}, w_{2} \in \Gamma_{m} \cap \Gamma_{m+1}, w_{5} \in \Gamma_{m+1}$ and since $\Gamma$ is of type $(m ; 2,3,2)$, we have $w_{5} \in \Gamma_{m+1} \cap \Gamma_{m+2}$.

Let $e_{5}$ be the terminal edge of label $m+1$ at $w_{5}$, and $a_{55}, b_{55}$ the internal edges (possibly terminal edges) of label $m+2$ at $w_{5}$ such that $a_{55}, e_{5}, b_{55}$ are consecutive edges at $w_{5}$. Since $a_{44}, b_{44}$ are oriented from $w_{4}$ to $w_{5}$, by Assumption 2 
(4) the two edges $a_{55}, b_{55}$ are oriented outward at $w_{5}$,

(5) neither $a_{55}$ nor $b_{55}$ is a terminal edge.

Let $E$ be the 2-angled disk of $\Gamma_{m+1}$ in $D_{1}$ with $\partial E=a_{44} \cup b_{44}$.

Claim 2. If $e_{5} \subset E$, then $w(\Gamma \cap \operatorname{Int} E) \geq 1$, otherwise $w\left(\Gamma \cap\left(\operatorname{Int} D_{1}-E\right)\right) \geq$ 1 .

Proof of Claim 2. If $e_{5} \subset E$, then (4) and (5) imply that $w(\Gamma \cap \operatorname{Int} E) \geq 1$ by IO-Calculation with respect to $\Gamma_{m+2}$ in $E$.

If $e_{5} \not \subset E$, then considering as $F=C l\left(D_{1}-E\right)$ and $k=m+2$ in the example of IO-Calculation in Section 6, we have $w\left(\Gamma \cap\left(\operatorname{Int} D_{1}-E\right)\right) \geq 1$. Hence Claim 2 holds.

Claim 3. $w\left(\Gamma \cap \operatorname{Int} D_{1}\right)=3, w\left(\Gamma \cap \operatorname{Int} D_{2}\right)=0$, and $w\left(\Gamma \cap \operatorname{Int} D_{3}\right)=1$.

Proof of Claim 3. Since $E \subset \operatorname{Int} D_{1}$, we have $w\left(\Gamma \cap \operatorname{Int} D_{1}\right) \geq 3$ by Claim 2 . By Claim 1, we have $w\left(\Gamma \cap \operatorname{Int} D_{3}\right) \geq 1$.

Suppose that $w\left(\Gamma \cap \operatorname{Int} D_{1}\right)>3$ or $w\left(\Gamma \cap \operatorname{Int} D_{2}\right)>0$ or $w\left(\Gamma \cap \operatorname{Int} D_{3}\right)>1$. Since $\Gamma$ is of type $(2,3,2)$, we have $w(\Gamma)=7$. Since the skew $\theta$-curve in $\Gamma_{m+1}$ contains exactly three white vertices, $7=w(\Gamma)=3+w\left(\Gamma \cap \operatorname{Int} D_{1}\right)+w\left(\Gamma \cap \operatorname{Int} D_{2}\right)+w\left(\Gamma \cap \operatorname{Int} D_{3}\right)>3+3+0+1=7$. This is a contradiction. Hence Claim 3 holds.

Claim 4. $e_{2}^{\prime \prime}=e_{3}^{\prime \prime}$ and $e_{2}^{\prime \prime \prime} \cap e_{3}^{\prime \prime \prime}$ contains a white vertex. Moreover the chart $\Gamma$ contains the pseudo chart as shown in Fig. 17(b).

Proof of Claim 4. We can show that the edge $e_{2}^{\prime \prime}$ is not a terminal edge by Assumption 2. Since $w\left(\Gamma \cap \operatorname{Int} D_{2}\right)=0$ by Claim 3, we have $e_{2}^{\prime \prime}=e_{3}^{\prime \prime}$.

By (1) and (3), both edges $e_{2}^{\prime \prime \prime}, e_{3}^{\prime \prime \prime}$ contain white vertices $w^{\prime}, w^{\prime \prime}$ different from $w_{2}, w_{3}$ respectively. Since $w\left(\Gamma \cap \operatorname{Int} D_{3}\right)=1$ by Claim 3 , the white vertices $w^{\prime}, w^{\prime \prime}$ are the same vertex. Hence $e_{2}^{\prime \prime \prime} \cap e_{3}^{\prime \prime \prime}$ contains a white vertex, say $w_{6}$. Moreover there exists a terminal edge of label $m+2$ at $w_{6}$, i.e. $w_{6}$ is a BW-vertex with respect to $\Gamma_{m+2}$. Hence the chart $\Gamma$ contains the pseudo chart as shown in Fig. 17(b). Thus Claim 4 holds.

Claim 5. The edge $e_{2}^{\prime}$ is not a terminal edge.

Proof of Claim 5. Let $D^{\prime}$ be the 3 -angled disk of $\Gamma_{m+2}$ with $\partial D^{\prime}=$ $e_{2}^{\prime \prime} \cup e_{2}^{\prime \prime \prime} \cup e_{3}^{\prime \prime \prime}$ and $w\left(\Gamma \cap \operatorname{Int} D^{\prime}\right)=0$ (see Fig. $17(\mathrm{~b})$ ).

If $e_{2}^{\prime}$ is a terminal edge, then $w\left(\Gamma \cap \operatorname{Int} D^{\prime}\right) \geq 1$ by Triangle Lemma (Lemma 6.1). This is a contradiction. Hence edge $e_{2}^{\prime}$ is not a terminal edge. Thus Claim 5 holds.

By Claim 2 and the first equation of Claim 3, we have the following claim:

Claim 6. If $e_{5} \subset E$, then $w(\Gamma \cap \operatorname{Int} E)=1$ and $w\left(\Gamma \cap\left(\operatorname{Int} D_{1}-E\right)\right)=0$, otherwise $w\left(\Gamma \cap\left(\operatorname{Int} D_{1}-E\right)\right)=1$.

If $e_{5} \subset E$, then let $w_{7}$ be the white vertex in $\operatorname{Int} E$, otherwise let $w_{7}$ be the white vertex in $\operatorname{Int} D_{1}-E$. 
Now we are ready to prove Lemma 7.3 . There are two cases: (i) $e_{5} \not \subset E$, (ii) $e_{5} \subset E$.

Case (i). By $e_{5} \not \subset E$, the chart $\Gamma$ contains the pseudo chart as shown in Fig. 17(a). We shall show that $e_{2}^{\prime}=e_{3}^{\prime}$ and $a_{55} \cap b_{55} \ni w_{7}$.

By Claim 5, the edge $e_{2}^{\prime}$ is not a terminal edge. Thus by (2),(4) and Claim 6, we have $e_{2}^{\prime}=e_{3}^{\prime}$ or $e_{2}^{\prime} \ni w_{7}$. Similarly by (2),(4),(5) and Claim 6, we can show that $\left(a_{55}=e_{3}^{\prime}\right.$ or $\left.a_{55} \ni w_{7}\right)$ and $\left(b_{55}=e_{3}^{\prime}\right.$ or $\left.b_{55} \ni w_{7}\right)$. Therefore $\left(e_{2}^{\prime}=e_{3}^{\prime}, a_{55} \ni w_{7}, b_{55} \ni w_{7}\right)$ or $\left(e_{2}^{\prime} \ni w_{7}, a_{55}=e_{3}^{\prime}, b_{55} \ni w_{7}\right)$ or $\left(e_{2}^{\prime} \ni w_{7}\right.$, $\left.a_{55} \ni w_{7}, b_{55}=e_{3}^{\prime}\right)$.

For the second case and third case, the graph $\Gamma_{m+2}$ contains the graph as shown in Fig. 17(c). However by Lemma 3.3, the graph $\Gamma_{m+2}$ does not contain the graph as shown in Fig. 17(c). Thus the second case and third case do not occur. Hence $e_{2}^{\prime}=e_{3}^{\prime}, a_{55} \cap b_{55} \ni w_{7}$.

Case (ii). By Claim 6, we have $w(\Gamma \cap \operatorname{Int} E)=1$. Thus by Lemma 7.2, the disk $E$ contains the pseudo chart as shown in Fig. 16 (see Fig. 17(d)). Hence $a_{55} \cap b_{55} \ni w_{7}$.

Now, the edge $e_{3}^{\prime}$ is oriented inward at $w_{3}$ but not middle at $w_{3}$. Thus by Assumption 2, the edge $e_{3}^{\prime}$ is not a terminal edge. Hence by Claim 5, we have $e_{2}^{\prime}=e_{3}^{\prime}$.

Let $e^{\prime}$ be an internal edge (possibly terminal edge) of label $m+2$ at $w_{5}$ in $C l\left(D_{1}-E\right)$. Since $w\left(\Gamma \cap\left(\operatorname{Int} D_{1}-E\right)\right)=0$ by Claim 6 , the condition $e_{2}^{\prime}=e_{3}^{\prime}$ implies that the edge $e^{\prime}$ is a terminal edge.

Hence by Lemma 7.1(b) there exists a special oval of label $m+2$ by $\mathrm{C}$-moves without changing the type of charts. Thus by X-change Lemma (Lemma 7.1(a)), the chart $\Gamma$ is $\mathrm{C}$-move equivalent to a minimal chart of type $(m ; 2,3,2)$ containing the pseudo chart as shown in Fig. 17(a).

Therefore, we complete the proof of Lemma 7.3 .

Similarly by applying several times of Lemma 7.1, we can show the following lemma.

Lemma 7.4 Let $\Gamma$ be a minimal chart of type $(m ; 2,3,2)$. If $\Gamma$ contains the pseudo chart as shown in Fig. $7(b)$, then the chart $\Gamma$ is $C$-move equivalent to a minimal chart of type $(m ; 2,3,2)$ containing the pseudo chart as shown in Fig. 17 (a). Moreover $e_{2}^{\prime}=e_{3}^{\prime}$ and $a_{55} \cap b_{55}$ contains a white vertex different from $w_{5}$.

\section{Disk Lemma}

In this section we shall show that neither $\Gamma_{m+1}$ nor $\Gamma_{m+2}$ contains an oval for any minimal chart $\Gamma$ of type $(m ; 2,3,2)$.

Let $\Gamma$ be a chart, and $D$ a disk. Let $\alpha$ be a simple arc in $\partial D$, and $\gamma$ a simple arc in an internal edge of label $k$. The simple arc $\gamma$ is called a $(D, \alpha)$-arc of label $k$ provided that $\partial \gamma \subset \operatorname{Int} \alpha$ and $\operatorname{Int} \gamma \subset \operatorname{Int} D$. If there is no $(D, \alpha)$-arc in $\Gamma$, then the chart $\Gamma$ is said to be $(D, \alpha)$-arc free. 


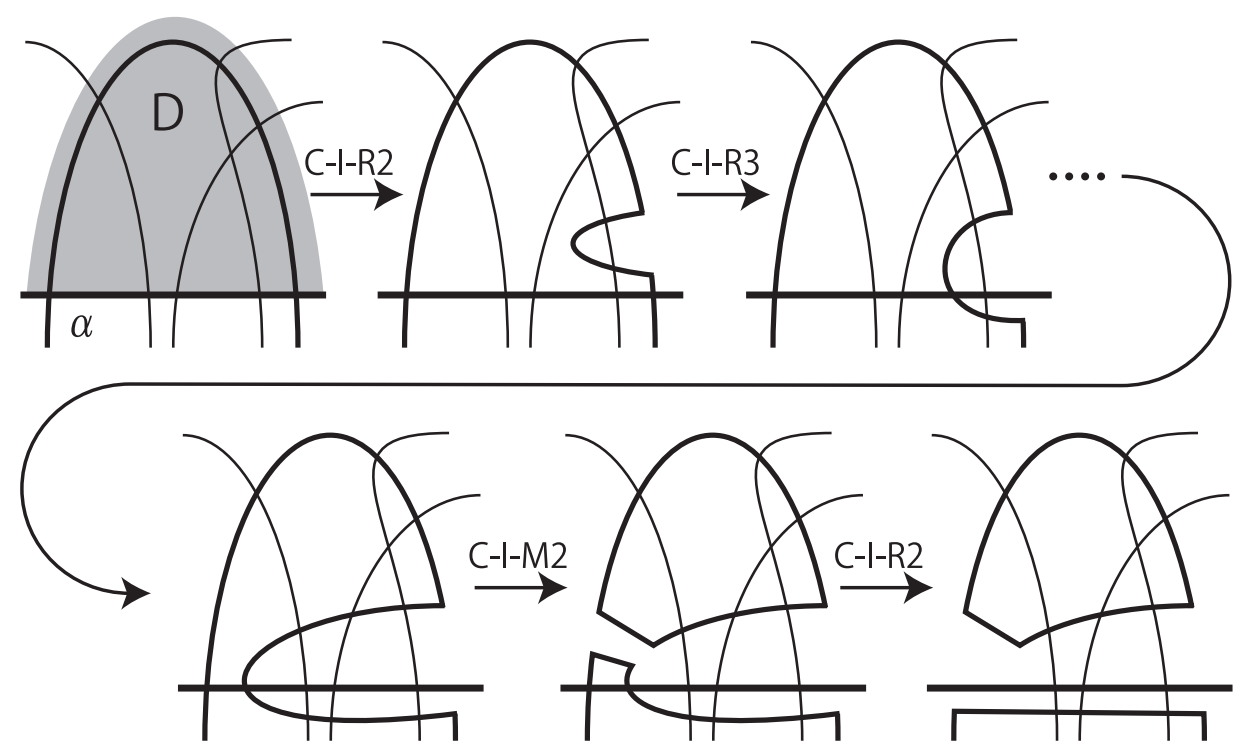

Figure 18: The gray region is the disk $D$.

Lemma 8.1 (New Disk Lemma) ([13, Lemma 7.1], cf. [6, Lemma 3.2]) Let $\Gamma$ be a chart and $D$ a disk whose interior does not contain a white vertex nor a black vertex of $\Gamma$. Let $\alpha$ be a simple arc in $\partial D$ such that Int $\alpha$ does not contain a white vertex nor a black vertex of $\Gamma$. Let $V$ be a regular neighborhood of $\alpha$. Suppose that the arc $\alpha$ is contained in an internal edge of some label $k$ of $\Gamma$. Then by applying C-I-M2 moves, $C$-I-R2 moves, and $C$-I-R3 moves in $V$, there exists a $(D, \alpha)$-arc free chart $\Gamma^{\prime}$ obtained from the chart $\Gamma$ keeping $\alpha$ fixed (see Fig. 18).

Lemma 8.2 ([9, Lemma 8.2]) Let $\Gamma$ be a chart, e an internal edge of label $m$, and $w_{1}, w_{2}$ the white vertices in e. Suppose $w_{1} \in \Gamma_{m-1}$ and $w_{2} \in \Gamma_{m+1}$. Then for any neighborhood $V$ of the edge $e$, there exists a chart $\Gamma^{\prime}$ obtained from the chart $\Gamma$ by $C$-I-R2 moves, $C$-I-R3 moves and $C$-I-R4 moves in $V$ keeping $\Gamma_{m-1} \cup \Gamma_{m} \cup \Gamma_{m+1}$ fixed such that the edge e does not contain any crossings.

Lemma 8.3 ([11, Lemma 7.1]) Let $G$ be one of 12 graphs as shown in Fig. 3 and Fig. 4 in [11]. If for any minimal chart $\Gamma$ of type $(m ; 2,3,2)$, the graph $\Gamma_{m+1}$ does not contain the graph $G$, then the graph $\Gamma_{m+2}$ does not contain the graph $G$.

Since the four graphs as shown in Fig. 4 and Fig. 5 are four graphs in the 12 graphs of the above lemma, we have the following lemma:

Lemma 8.4 Let $G$ be one of four graphs as shown in Fig. 4 and Fig. 5 . If for any minimal chart $\Gamma$ of type $(m ; 2,3,2)$, the graph $\Gamma_{m+1}$ does not contain the graph $G$, then the graph $\Gamma_{m+2}$ does not contain the graph $G$. 
Proposition 8.5 (cf. [10, Lemma 6.3]) Let $\Gamma$ be a minimal chart of type $(m ; 2,3,2)$. Then neither $\Gamma_{m+1}$ nor $\Gamma_{m+2}$ contains an oval.

Proof. Suppose that $\Gamma_{m+1}$ contains an oval. By Lemma 4.1. Lemma 7.3 and Lemma 7.4, the chart $\Gamma$ contains the pseudo chart as shown in Fig. 17(a), where $e_{2}^{\prime}, e_{3}^{\prime}, a_{55}, b_{55}$ are internal edges of label $m+2$ at $w_{2}, w_{3}, w_{5}, w_{5}$ respectively and

(1) $e_{2}^{\prime}=e_{3}^{\prime}$,

(2) $a_{55} \cap b_{55}$ contains a white vertex different from $w_{5}$, say $w_{7}$.

Since $w_{1}, w_{2}, w_{3}, w_{4}, w_{5} \in \Gamma_{m+1}, w_{6}, w_{7} \in \Gamma_{m+2}$ and since $\Gamma$ is of type $(m ; 2,3,2)$, we have

(3) $\Gamma_{m+3}$ contains exactly two white vertices $w_{6}, w_{7}$.

By (1), the union $e_{2}^{\prime} \cup e_{2}^{\prime \prime}$ bounds a 2-angled disk of $\Gamma_{m+2}$. Let $E^{\prime}$ be the 2-angled disk of $\Gamma_{m+2}$ with $\partial E^{\prime}=e_{2}^{\prime} \cup e_{2}^{\prime \prime}$ and $\operatorname{Int} E^{\prime} \ni w_{1}$.

Claim 1. Int $E^{\prime}$ contains exactly one white vertex $w_{1}$.

Proof of Claim 1. It is possible that Int $E^{\prime}$ contains $w_{1}, w_{4}, w_{5}, w_{7}$.

We shall show $w_{7} \notin \operatorname{Int} E^{\prime}$. If $w_{7} \in \operatorname{Int} E^{\prime}$, then the two white vertices $w_{6}$ and $w_{7}$ of $\Gamma_{m+3}$ are separated by the simple closed curve $\partial E^{\prime}$ of label $m+2$. Thus by (3) there exists a connected component of $\Gamma_{m+3}$ with exactly one white vertex. This contradicts Lemma 3.2. Hence $w_{7} \notin \operatorname{Int} E^{\prime}$.

We shall show that neither $w_{4}$ nor $w_{5}$ is contained in $\operatorname{Int} E^{\prime}$. If one of $w_{4}, w_{5}$ is contained in $\operatorname{Int} E^{\prime}$, then both of $w_{4}$ and $w_{5}$ are contained in $\operatorname{Int} E^{\prime}$, because $\partial E^{\prime} \subset \Gamma_{m+2}$ and $w_{4}, w_{5} \in a_{44} \subset \Gamma_{m+1}$. Moreover $w_{5} \in a_{55} \cap b_{55}$ implies $a_{55} \cup b_{55} \subset \operatorname{Int} E^{\prime}$. Thus $w_{7} \in a_{55} \cap b_{55}$ by (2) implies $w_{7} \in \operatorname{Int} E^{\prime}$. This is a contradiction. Hence neither $w_{4}$ nor $w_{5}$ is contained in $\operatorname{Int} E^{\prime}$. Therefore Int $E^{\prime}$ contains exactly one white vertex $w_{1}$. Thus Claim 1 holds.

Let $e_{1}^{\prime}, e_{1}^{\prime \prime}$ be the internal edges of label $m+1$ at $w_{1}$ (see Fig. $\left.17(\mathrm{a})\right)$. Then the arc $e_{1}^{\prime} \cup e_{1}^{\prime \prime}$ separates the disk $E^{\prime}$ into two disks. Let $F_{1}$ be one of the two disks with $\partial F_{1} \supset e_{2}^{\prime}$. By Claim 1, we have

(4) $w\left(\Gamma \cap \operatorname{Int} F_{1}\right)=0$.

Let $e_{1}, e_{2}$ be the internal edges of label $m$ connecting $w_{1}$ and $w_{4}$.

Claim 2. We can assume that for each $i=1,2$ the intersection $e_{i} \cap e_{2}^{\prime}$ is one point by C-moves in a neighborhood of $F_{1}$ keeping $\Gamma_{m+1} \cup \Gamma_{m+2} \cup \Gamma_{m+3}$ fixed (see Fig. 19(a)).

Proof of Claim 2. Since $w_{4}$ is contained in the outside of $E^{\prime}$ by Claim 1, for each $i=1,2$ the intersection $e_{i} \cap E^{\prime}$ consists of an arc with $w_{1}$ and proper arcs of $E^{\prime}$. Since $F_{1}$ is obtained from $E^{\prime}$ by cutting along the arc $e_{1}^{\prime} \cup e_{1}^{\prime \prime}$ in $\Gamma_{m+1}$,

(5) $e_{i} \cap F_{1}=e_{i} \cap E^{\prime}$ consists of an arc with $w_{1}$ and $\left(F_{1}, e_{2}^{\prime}\right)$-arcs of label $m$ (see Fig 19(b)). 
Let $N$ be a regular neighborhood of the terminal edge $e$ of label $m+1$ at $w_{1}$ in $F_{1}$. Then $\widetilde{F}_{1}=C l\left(F_{1}-N\right)$ is a disk. By (4), we can apply New Disk Lemma (Lemma 8.1 for the disk $\widetilde{F_{1}}$ so that the chart $\Gamma$ is $\left(\widetilde{F_{1}}, e_{2}^{\prime}\right)$-arc free. Thus by (5), $e_{i} \cap F_{1}$ is an arc with $w_{1}$. Hence $e_{i} \cap e_{2}^{\prime}$ is one point. Thus Claim 2 holds.

By (3), we have $w_{7} \in \Gamma_{m+3}$. Hence by (2), the edge $a_{55}$ of label $m+2$ connects $w_{5} \in \Gamma_{m+1}$ and $w_{7} \in \Gamma_{m+3}$. Thus by Lemma 8.2 we can assume that

(6) the edge $a_{55}$ does not contain any crossing.

Let $F_{2}$ be the 2-angled disk of $\Gamma_{m+2}$ with $\partial F_{2}=a_{55} \cup b_{55}$ and $w_{6} \notin F_{2}$. Since $w_{7} \in \partial F_{2}$, we have

(7) $w\left(\Gamma_{m+3} \cap \operatorname{Int} F_{2}\right)=0$ by $(3)$,

(8) Int $F_{2}$ contains at most one white vertex $w_{4}$.

Let $e_{7}$ be the terminal edge of label $m+2$ at $w_{7}$.

Claim 3. $e_{7} \not \subset F_{2}$.

Proof of Claim 3. Suppose $e_{7} \subset F_{2}$. Then by (3), there exist two internal edges $e_{7}^{\prime}, e_{7}^{\prime \prime}$ (possibly terminal edges) of label $m+3$ at $w_{7}$ in $F_{2}$. Since both of the edges $a_{55}$ and $b_{55}$ of label $m+2$ are oriented from $w_{5}$ to $w_{7}$ (see Fig. 19(a)), both of $e_{7}^{\prime}$ and $e_{7}^{\prime \prime}$ are oriented outward at $w_{7}$. Moreover by Assumption 2, neither $e_{7}^{\prime}$ nor $e_{7}^{\prime \prime}$ is a terminal edge. Hence we have $w\left(\Gamma_{m+3} \cap \operatorname{Int} F_{2}\right) \geq 1$ by IO-Calculation with respect to $\Gamma_{m+3}$ in $F_{2}$. This contradicts (7). Thus $e_{7} \not \subset F_{2}$. Hence Claim 3 holds.

Now we are ready to prove Proposition 8.5. There are two cases: (i) $F_{2} \ni w_{4}$, (ii) $F_{2} \not \supset w_{4}$.

Case (i). First we shall show that for each $i=1,2$, the intersection $e_{i} \cap \partial F_{2}$ is one point by C-moves in a neighborhood of $F_{2}$ keeping $\Gamma_{m+1} \cup$ $\Gamma_{m+2} \cup \Gamma_{m+3}$ fixed (see Fig. 11(b)).

Since $w_{4} \in \operatorname{Int} F_{2}$ by the condition of Case (i), we have $a_{44} \cup b_{44} \subset F_{2}$. Let $E$ be the 2-angled disk of $\Gamma_{m+1}$ in $F_{2}$ with $\partial E=a_{44} \cup b_{44}$. By (8), we have

(9) $w\left(\Gamma \cap\left(\operatorname{Int} F_{2}-E\right)\right)=0$.

Since for each $i=1,2$ the edge $e_{i}$ connects the white vertex $w_{4} \operatorname{in} \operatorname{Int} F_{2}$ and the white vertex $w_{1}$ in the outside of $F_{2}$, the intersection $e_{i} \cap F_{2}$ consists of an arc with $w_{4}$ and proper arcs of $F_{2}$. Moreover $\partial E \subset \Gamma_{m+1}$ and (6) imply

(10) $e_{i} \cap F_{2}=e_{i} \cap C l\left(F_{2}-E\right)$,

(11) $e_{i} \cap F_{2}$ consists of an arc with $w_{4}$ and $\left(F_{2}, b_{55}\right)$-arcs of label $m$. 

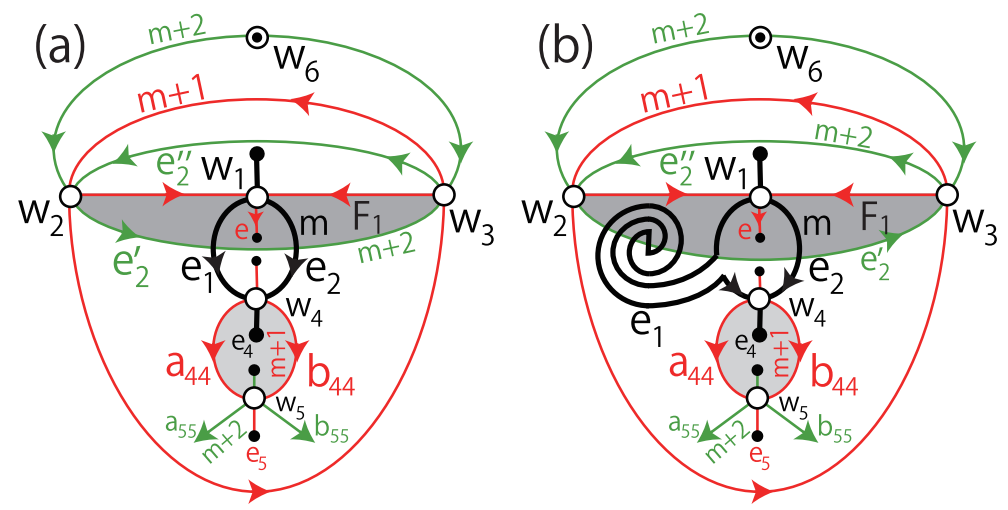

Figure 19: The dark gray regions are the disk $F_{1}$. The light gray regions are the disk $E$.

By (7) and Claim 3, there exists a terminal edge $e^{\prime}$ of label $m+3$ at $w_{7}$. Let $e^{\prime \prime}$ be the terminal edge of label $m+1$ at $w_{4}$, and $N^{\prime}$ a regular neighborhood of $E \cup e^{\prime} \cup e^{\prime \prime}$ in $F_{2}$. Then $\widetilde{F}_{2}=C l\left(F_{2}-N^{\prime}\right)$ is a disk. Let $\widetilde{b_{55}}=b_{55} \cap \widetilde{F_{2}}$. By $(9)$, we can apply New Disk Lemma (Lemma 8.1) for the disk $\widetilde{F_{2}}$ so that the chart $\Gamma$ is $\left(\widetilde{F_{2}}, \widetilde{b_{55}}\right)$-arc free. Thus there is no $\left(\widetilde{F_{2}}, \widetilde{b_{55}}\right)$-arc of label $m$, i.e. there is no $\left(F_{2}, b_{55}\right)$-arcs of label $m$. Thus by (10) and (11) for each $i=1,2$, the intersection $e_{i} \cap F_{2}$ is an arc with $w_{4}$. Therefore the chart $\Gamma$ contains the pseudo chart as shown in Fig. 11(b). However by Lemma 5.3, the chart $\Gamma$ is not minimal. This is a contradiction. Hence Case (i) does not occur.

Case (ii). Similarly we can show that $e_{i} \cap \partial F_{2}=\emptyset$ for each $i=1,2$ by C-moves in a neighborhood of $F_{2}$ keeping $\Gamma_{m+1} \cup \Gamma_{m+2} \cup \Gamma_{m+3}$ fixed. Thus the chart $\Gamma$ contains the pseudo chart as shown in Fig. 11(a). However by Lemma 5.3 , the chart $\Gamma$ is not minimal. This is a contradiction. Hence Case (ii) does not occur.

Therefore the graph $\Gamma_{m+1}$ does not contain an oval. By Lemma 8.4, the graph $\Gamma_{m+2}$ does not contain an oval. We complete the proof of Proposition 8.5

By Lemma 3.3 and Proposition 8.5, we have the following corollary:

Corollary 8.6 If there exists a minimal chart $\Gamma$ of type $(m ; 2,3,2)$, then each of $\Gamma_{m+1}$ and $\Gamma_{m+2}$ contains one of two graphs as shown in Fig. 5 .

\section{$9 \quad 3$-angled disks}

In this section, we investigate a minimal chart $\Gamma$ of type $(m ; 2,3,2)$ such that $\Gamma_{m+1}$ contains the graph as shown in Fig. 5(a).

Let $X$ be a set in a chart $\Gamma$. Let

$$
c(X)=\text { the number of crossings on } X \text {. }
$$


Let $D$ be a $k$-angled disk of $\Gamma_{m}$ for a minimal chart $\Gamma$. The pair of integers $(w(\Gamma \cap \operatorname{Int} D), c(\Gamma \cap \partial D))$ is called the local complexity with respect to $D$, denoted by $\ell c(D ; \Gamma)$. Let $\mathbb{S}$ be the set of all minimal charts each of which can be moved from $\Gamma$ by $\mathrm{C}$-moves in a regular neighborhood of $D$ keeping $\partial D$ fixed. The chart $\Gamma$ is said to be locally minimal with respect to $D$ if its local complexity with respect to $D$ is minimal among the charts in $\mathbb{S}$ with respect to the lexicographic order.

Let $\Gamma$ be a chart, and $D$ a $k$-angled disk of $\Gamma_{m}$. If any feeler of $D$ of label $m$ is a terminal edge, then $D$ is called a special $k$-angled disk.

Lemma 9.1 ([8, Theorem 1.2]) Let $\Gamma$ be a minimal chart. Let $D$ be a special 3-angled disk of $\Gamma_{m}$ such that $\Gamma$ is locally minimal with respect to $D$. If $w(\Gamma \cap \operatorname{Int} D) \leq 1$, then a regular neighborhood of $D$ contains one of the $R O$ families of the eight pseudo charts as shown in Fig. 20.


Figure 20: The 3-angled disks (g) and (h) have one feeler, the others do not have any feelers. $m$ is a label, $\varepsilon, \delta \in\{+1,-1\}$.

Lemma 9.2 Let $\Gamma$ be a minimal chart of type $(m ; 2,3,2)$. Suppose that $\Gamma_{m+1}$ contains the graph as shown in Fig. $5\left(\right.$ a). Let $D_{1}$ be a 3-angled disk of $\Gamma_{m+1}$ with $w\left(\Gamma_{m+1} \cap \operatorname{Int} D_{1}\right)=0$. If $w\left(\Gamma \cap \operatorname{Int} D_{1}\right) \leq 1$ and if $\Gamma$ is locally minimal with respect to $D_{1}$, then $\Gamma$ contains one of the $R O$-families of the three pseudo charts as shown in Fig. 21. 


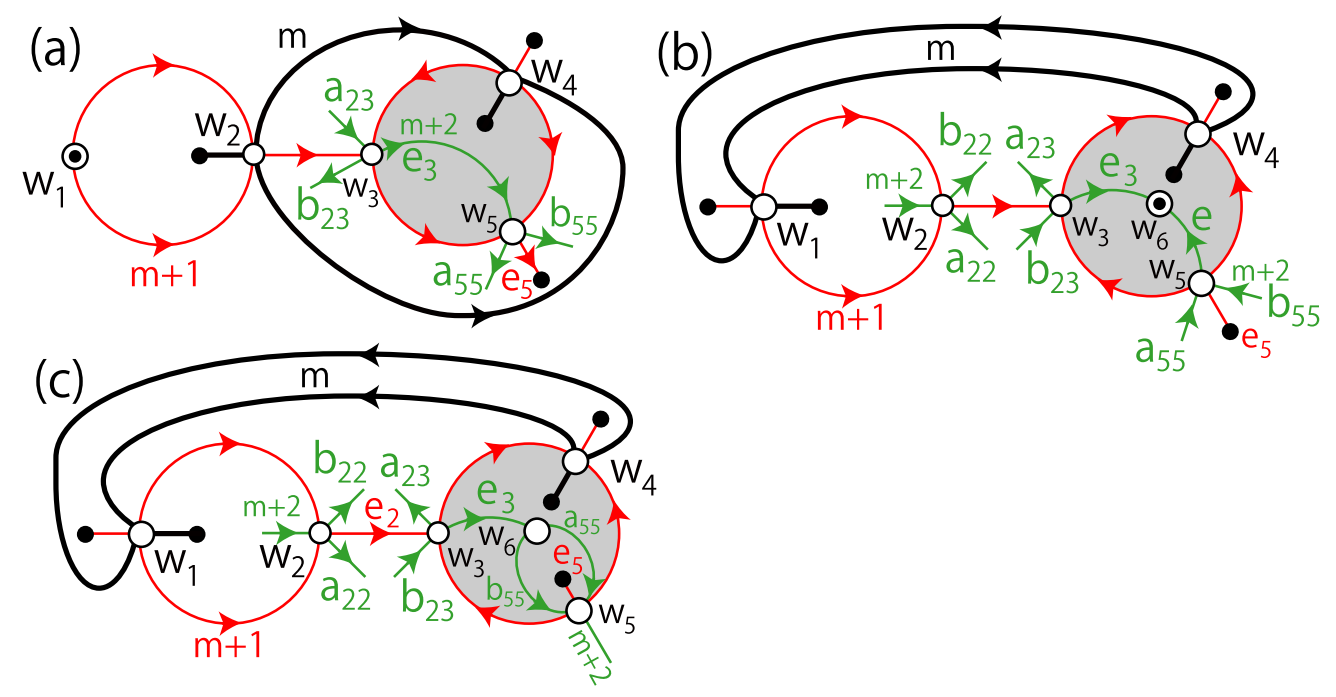

Figure 21: The gray regions are the disk $D_{1}$.

Proof. By Lemma 4.2, the chart $\Gamma$ contains one of the RO-families of the two pseudo charts as shown in Fig. 8(a),(b). We use the notations as shown in Fig. 8(a),(b), where

(1) $e_{3}$ is an internal edge (possibly terminal edge) of label $m+2$ oriented outward at $w_{3}$,

(2) $w_{3} \in \Gamma_{m+2}, w_{4} \in \Gamma_{m}$.

We shall show $w_{5} \in \Gamma_{m+2}$. Since $w_{5} \in \Gamma_{m+1}$, we have $w_{5} \in \Gamma_{m}$ or $w_{5} \in \Gamma_{m+2}$. Suppose $w_{5} \in \Gamma_{m}$. Since one of $w_{1}, w_{2}$ is in $\Gamma_{m}$, by (2) the graph $\Gamma_{m}$ contains at least three white vertices. This contradicts the fact that $\Gamma$ is of type $(m ; 2,3,2)$. Hence we have $w_{5} \in \Gamma_{m+2}$.

Since $w_{4} \in \Gamma_{m}$ and $w_{3}, w_{5} \in \Gamma_{m+2}$ by $(2)$, a regular neighborhood $N\left(D_{1}\right)$ of $D_{1}$ contains one of the RO-families of the five pseudo charts as shown in Fig. 20(a),(d),(e),(g),(h) by Lemma 9.1 .

Looking at Fig. 8(a),(b), we see that the terminal edge of label $m+1$ at $w_{4}$ is not contained in $D_{1}$. Thus $N\left(D_{1}\right)$ does not contain one of the RO-family of the pseudo chart as shown in Fig. 20(h).

Let $e_{5}$ be the terminal edge of label $m+1$ at $w_{5}$.

If $N\left(D_{1}\right)$ contains one of the RO-family of the pseudo chart as shown in Fig. 20(a), then $e_{5} \not \subset D_{1}$ and $e_{3} \ni w_{5}$. Thus by (1), the edge $e_{3}$ is oriented inward at $w_{5}$. Hence $\Gamma$ contains the pseudo chart as shown in Fig. 8(b). Thus $\Gamma$ contains the pseudo chart as shown in Fig. 21(a).

If $N\left(D_{1}\right)$ contains one of the RO-families of the two pseudo charts as shown in Fig. 20(d),(e), then $e_{5} \not \subset D_{1}$. Moreover there exists only one internal edge $e$ of label $m+2$ at $w_{5}$ in $D_{1}$ such that $e \cap e_{3}$ is a BW-vertex with respect to $\Gamma_{m+2}$. Thus by (1) and Lemma 3.1, the edge $e$ is oriented outward at $w_{5}$. Hence $\Gamma$ contains the pseudo chart as shown in Fig. 8(a). Thus $\Gamma$ contains the pseudo chart as shown in Fig. 21(b). 
If $N\left(D_{1}\right)$ contains one of the RO-family of the pseudo chart as shown in Fig. 20 (g), then $e_{5} \subset D_{1}$ and there exist two internal edges $a_{55}, b_{55}$ of label $m+2$ at $w_{5}$ in $D_{1}$ such that $e_{3} \cap a_{55} \cap b_{55}$ is a white vertex. Thus by (1) and Assumption 2, the edges $a_{55}, b_{55}$ are oriented inward at $w_{5}$. Hence $\Gamma$ contains the pseudo chart as shown in Fig. 8(a). Thus $\Gamma$ contains the pseudo chart as shown in Fig. 21(c).

\section{Case of the graph as shown in Fig. 5(a)}

In this section we shall show that neither $\Gamma_{m+1}$ nor $\Gamma_{m+2}$ contains the graph as shown in Fig. 5(a) for any minimal chart $\Gamma$ of type $(m ; 2,3,2)$.

Let $\Gamma$ be a chart, and $m$ a label of $\Gamma$. A loop is a simple closed curve in $\Gamma_{m}$ with exactly one white vertex (possibly with crossings).

Lemma 10.1 ([9, Theorem 1.1]) There is no loop in any minimal chart with exactly seven white vertices.

Lemma 10.2 Let $\Gamma$ be a chart of type $(m ; 2,3,2)$. If $\Gamma$ contains the pseudo chart as shown in Fig. $8(a)$, then $\Gamma$ is not minimal.

Proof. Suppose that $\Gamma$ is minimal. We use the notations as shown in Fig. 8(a), where

(1) $w_{2} \in \Gamma_{m+1} \cap \Gamma_{m+2}$,

(2) $a_{22}, b_{22}, a_{23}$ are internal edges (possibly terminal edges) of label $m+2$ oriented outward at $w_{2}, w_{2}, w_{3}$ but not middle at $w_{2}, w_{2}, w_{3}$, respectively,

(3) $b_{23}$ is an internal edge (possibly terminal edge) of label $m+2$ middle at $w_{3}$.

By (2) and Assumption 2.

(4) none of $a_{22}, b_{22}, a_{23}$ are terminal edges.

Let $D_{1}$ be the 3 -angled disk of $\Gamma_{m+1}$ with $\partial D_{1} \ni w_{3}, w_{4}, w_{5}$ and $D_{1} \supset e_{3}$ (see Fig. $8($ a)). We can assume that

(5) $\Gamma$ is locally minimal with respect to $D_{1}$.

Claim 1. $w\left(\Gamma \cap \operatorname{Int} D_{1}\right) \geq 1$.

Proof of Claim 1. Suppose that $w\left(\Gamma \cap \operatorname{Int} D_{1}\right)=0$. Since $\Gamma_{m+1}$ contains the graph as shown in Fig. 5 (a), by (5) and Lemma 9.2 the chart $\Gamma$ contains one of the RO-family of the pseudo chart as shown in Fig. 21(a). Thus $w_{2} \in \Gamma_{m}$. This contradicts (1). Hence we have $w\left(\Gamma \cap \operatorname{Int} D_{1}\right) \geq 1$. Thus Claim 1 holds. 
Let $D_{2}$ be the 2-angled disk of $\Gamma_{m+1}$ with $\partial D_{2} \ni w_{1}, w_{2}$ and $D_{1} \cap D_{2}=\emptyset$ (see Fig. 8(a)).

Claim 2. $w\left(\Gamma \cap\left(S^{2}-\left(D_{1} \cup D_{2}\right)\right)\right) \geq 1$.

Proof of Claim 2. Suppose that $w\left(\Gamma \cap\left(S^{2}-\left(D_{1} \cup D_{2}\right)\right)\right)=0$. Since $a_{22}, b_{22}, a_{23}$ are oriented outward at $w_{2}, w_{2}, w_{3}$ respectively by (2), and since none of $a_{22}, b_{22}, a_{23}$ are terminal edges by (4), we have $a_{22}=b_{23}$ and $b_{22} \cap a_{23} \ni$ $w_{5}$ (see Fig. 22(a)). Thus the three white vertices $w_{2}, w_{3}, w_{5}$ are contained in the simple closed curve $C=a_{22} \cup b_{22} \cup a_{23}$ of $\Gamma_{m+2}$ with $w(C)=3$. Hence by Corollary 8.6, the graph $\Gamma_{m+2}$ contains the graph as shown in Fig. 5(a). Thus by Lemma 4.4. the simple closed curve $C$ contains one white vertex in $\Gamma_{m+3}$ (see Fig. 9 $(\mathrm{a}),(\mathrm{b})$ ). However all of the white vertices $w_{2}, w_{3}, w_{5}$ in $C$ are contained in $\Gamma_{m+1} \cap \Gamma_{m+2}$. This is a contradiction. Hence $w\left(\Gamma \cap\left(S^{2}-\left(D_{1} \cup D_{2}\right)\right)\right) \geq 1$. Thus Claim 2 holds.

Claim 3. $w\left(\Gamma \cap \operatorname{Int} D_{1}\right)=1$ and $w\left(\Gamma \cap\left(S^{2}-\left(D_{1} \cup D_{2}\right)\right)\right)=1$.

Proof of Claim 3. Since $D_{2}$ is a 2-angled disk, we have $w\left(\Gamma \cap D_{2}\right) \geq 2$. Since $D_{1}$ is a 3 -angled disk, we have $w\left(\Gamma \cap \partial D_{1}\right)=3$. Thus

$$
w\left(\Gamma \cap D_{1}\right)=w\left(\Gamma \cap \partial D_{1}\right)+w\left(\Gamma \cap \operatorname{Int} D_{1}\right)=3+w\left(\Gamma \cap \operatorname{Int} D_{1}\right) .
$$

Hence

$$
\begin{aligned}
7=w(\Gamma) & =w\left(\Gamma \cap D_{1}\right)+w\left(\Gamma \cap D_{2}\right)+w\left(\Gamma \cap\left(S^{2}-\left(D_{1} \cup D_{2}\right)\right)\right) \\
& \geq 3+w\left(\Gamma \cap \operatorname{Int} D_{1}\right)+2+w\left(\Gamma \cap\left(S^{2}-\left(D_{1} \cup D_{2}\right)\right)\right) .
\end{aligned}
$$

Thus

$$
2 \geq w\left(\Gamma \cap \operatorname{Int} D_{1}\right)+w\left(\Gamma \cap\left(S^{2}-\left(D_{1} \cup D_{2}\right)\right)\right) .
$$

Hence Claim 1 and Claim 2 imply Claim 3.

By Claim 3, there exists a white vertex in $\operatorname{Int} D_{1}$, say $w_{6}$, and there exists a white vertex in $S^{2}-\left(D_{1} \cup D_{2}\right)$, say $w_{7}$. Since $\Gamma$ is of type $(m ; 2,3,2)$ and since $w_{1}, w_{2}, w_{3}, w_{4}, w_{5} \in \Gamma_{m+1}$, we have

(6) the graph $\Gamma_{m+3}$ contains only two white vertices $w_{6}, w_{7}$.

Since $w\left(\Gamma \cap \operatorname{Int} D_{1}\right)=1$ by Claim 3, the chart $\Gamma$ contains one of the RO-families of the two pseudo charts as shown in Fig. 21(b),(c) by (5) and Lemma 9.2. Hence there are two cases.

Case (i). Suppose that the chart $\Gamma$ contains one of the RO-family of the pseudo chart as shown in Fig. 21(b). We use the notations as shown in Fig. 21(b), where

(7) both internal edges $e_{3}, e$ of label $m+2$ are oriented inward at $w_{6}$, and $a_{55}, b_{55}$ are internal edges (possibly terminal edges) of label $m+2$ at $w_{5}$ not middle at $w_{5}$. Thus by Assumption 2 ,

(8) neither $a_{55}$ nor $b_{55}$ is a terminal edge. 
By Corollary 8.6, the graph $\Gamma_{m+2}$ contains one of the two graphs as shown in Fig. 5. Hence there are two cases.

If $\Gamma_{m+2}$ contains the graph as shown in Fig. 5(a), then the white vertex $w_{6}$ must contain the simple closed curve $C$ of label $m+2$ with $w(C)=3$. Thus by (8), we have $b_{55}=a_{23}$ and $C=b_{55} \cup e_{3} \cup e$. Hence the edge $b_{23}$ is a terminal edge, $a_{22} \cap b_{22} \cap a_{55} \ni w_{7}$ (see Fig. $22(\mathrm{~b})$ ).

Since both of $a_{22}$ and $b_{22}$ of label $m+2$ are oriented from $w_{2}$ to $w_{7}$ by (2), there exist two internal edges $e_{7}^{\prime}, e_{7}^{\prime \prime}$ (possibly terminal edges) of label $m+3$ oriented outward at $w_{7}$ but not middle at $w_{7}$. Thus by Assumption 2 , neither $e_{7}^{\prime}$ nor $e_{7}^{\prime \prime}$ is a terminal edge. Hence by Lemma 10.1 and (6), we have $e_{7}^{\prime} \cap e_{7}^{\prime \prime} \ni w_{6}$. Thus the two edges $e_{7}^{\prime}, e_{7}^{\prime \prime}$ of label $m+3$ are oriented inward at $w_{6}$. However by (7) there exist four edges oriented inward at $w_{6}$. This contradicts Condition (iii) of the definition of a chart. Hence $\Gamma_{m+2}$ does not contain the graph as shown in Fig. 5(a).

If $\Gamma_{m+2}$ contains the graph as shown in Fig. 5(b), then the BW-vertex $w_{6}$ with respect to $\Gamma_{m+2}$ is not contained in any simple closed curve of label $m+2$ (Fig. 21(b)). Hence both of $a_{23} \cup b_{23}$ and $a_{55} \cup b_{55}$ are simple closed curves. Thus by Lemma 4.4, any simple closed curve of label $m+2$ does not contain middle arcs at any white vertex (see Fig. 9(c),(d)). Hence the edge $b_{23}$ is not middle at $w_{3}$. This contradicts (3). Thus $\Gamma_{m+2}$ does not contain the graph as shown in Fig. 5(b).

Hence neither two cases occur. Thus Case (i) does not occur.

Case (ii). Suppose that the chart $\Gamma$ contains one of the RO-family of the pseudo chart as shown in Fig. 21(c). We use the notations as shown in Fig. 21(c), where

(9) $a_{55} \cup b_{55}$ is a simple closed curve.

By Corollary 8.6, the graph $\Gamma_{m+2}$ contains one of the two graphs as shown in Fig. 5. Hence there are two cases.

If $\Gamma_{m+2}$ contains the graph as shown in Fig. 5(a), then there exists a simple closed curve $C$ of label $m+2$ with $w(C)=3$. Thus by (9), $w_{5} \notin C$ and $w_{6} \notin C$. Hence $C \ni w_{2}, w_{3}, w_{7}$ and $C=a_{22} \cup b_{22} \cup a_{23}$. Moreover there exist terminal edges of label $m+2$ at $w_{2}, w_{7}$ respectively.

Let $D_{3}$ be the 3 -angled disk of $\Gamma_{m+2}$ with $\partial D_{3}=C$ which contains the internal edge $e_{2}$ of label $m+1$ connecting $w_{2}, w_{3}$. By (6) and Corollary of Triangle Lemma (Corollary 6.2), we have $w\left(\Gamma \cap \operatorname{Int} D_{3}\right) \geq 1$. Thus there exists a white vertex in $\operatorname{Int} D_{3}$ different from $w_{7}$. Thus the condition $\operatorname{Int} D_{3} \subset$ $S^{2}-\left(D_{1} \cup D_{2}\right)$ implies $w\left(\Gamma \cap\left(S^{2}-\left(D_{1} \cup D_{2}\right)\right)\right) \geq 2$. This contradicts Claim 3. Hence $\Gamma_{m+2}$ does not contain the graph as shown in Fig. 5 (a).

If $\Gamma_{m+2}$ contains the graph as shown in Fig. 5(b), then by (9) one of the edges $a_{23}, b_{23}$ is a terminal edge. Since $a_{23}$ is not a terminal edge by (4), the edge $b_{23}$ is a terminal edge. Hence $a_{22} \cap b_{22} \cap a_{23} \ni w_{7}$. However by (2), all of the three edges $a_{22}, b_{22}, a_{23}$ are edges of label $m+2$ oriented inward at $w_{7}$. This contradicts Condition (iii) of the definition of a chart. Hence $\Gamma_{m+2}$ does not contain the graph as shown in Fig. 5(b). 
Thus neither two cases occur. Hence Case (ii) does not occur.

Therefore neither Case (i) nor Case (ii) occurs. Hence $\Gamma$ is not minimal. We complete the proof of Lemma 10.2 .
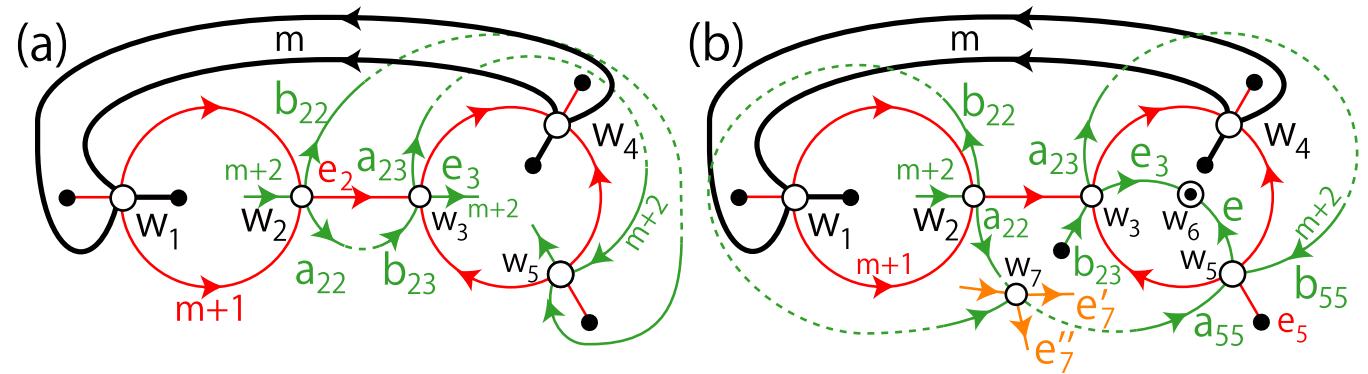

Figure 22: (a) There is a simple closed curve $a_{22} \cup b_{22} \cup a_{23}$ in $\Gamma_{m+2}$. The graph $\Gamma_{m+2}$ contains the graph as shown in Fig. 5)(a).

Proposition 10.3 Let $\Gamma$ be a minimal chart of type $(m ; 2,3,2)$. Then neither of $\Gamma_{m+1}$ nor $\Gamma_{m+2}$ contains the graph as shown in Fig. $5(a)$.

Proof. Suppose that $\Gamma_{m+1}$ contains the graph as shown in Fig. 5(a). By Lemma 4.2 and Lemma 10.2 , the chart $\Gamma$ contains one of the RO-family of the pseudo chart as shown in Fig. 8(b). We use the notations as shown in Fig. 8(b), where

(1) $w_{2} \in \Gamma_{m}$.

Moreover, $b_{23}$ is an internal edge (possibly terminal edge) of label $m+2$ at $w_{3}$ but not middle at $w_{3}$. By Assumption 2,

(2) $b_{23}$ is not a terminal edge.

Let $D_{1}$ be the 3 -angled disk of $\Gamma_{m+1}$ with $\partial D_{1} \ni w_{3}, w_{4}, w_{5}$ and $D_{1} \supset e_{3}$ (see Fig. 8(b)).

Claim 1. $w\left(\Gamma \cap \operatorname{Int} D_{1}\right) \geq 2$.

Proof of Claim 1. Suppose that $w\left(\Gamma \cap \operatorname{Int} D_{1}\right) \leq 1$. We can assume that $\Gamma$ is locally minimal with respect to $D_{1}$. Thus (1) and Lemma 9.2 imply that the chart $\Gamma$ contains one of the RO-family of the pseudo chart as shown in Fig. 21(a). Since a neighborhood of $D_{1}$ contains the pseudo chart as shown in Fig. 13(a), we can apply Triangle Lemma (Lemma 6.1) so that $w\left(\Gamma \cap \operatorname{Int} D_{1}\right) \geq 1$. Hence $w\left(\Gamma \cap \operatorname{Int} D_{1}\right)=1$.

Let $w_{6}$ be the white vertex in $\operatorname{Int} D_{1}$. Since $w_{1}, w_{2}, w_{3}, w_{4}, w_{5} \in \Gamma_{m+1}$ and since $\Gamma$ is of type $(m ; 2,3,2)$, we have $w_{6} \in \Gamma_{m+2} \cap \Gamma_{m+3}$. Hence there exists a connected component of $\Gamma_{m+2}$ with only one white vertex $w_{6}$. This contradicts Lemma 3.2. Thus $w\left(\Gamma \cap \operatorname{Int} D_{1}\right) \geq 2$. Hence Claim 1 holds.

Let $D_{2}$ be the 2-angled disk of $\Gamma_{m+1}$ with $\partial D_{2} \ni w_{1}, w_{2}$ and $D_{1} \cap D_{2}=\emptyset$ (see Fig. 8(b)). 
Claim 2. $w\left(\Gamma \cap \operatorname{Int} D_{2}\right)=0$ and $w\left(\Gamma \cap\left(S^{2}-\left(D_{1} \cup D_{2}\right)\right)\right)=0$.

Proof of Claim 2. Since $D_{1}$ is a 3 -angled disk, by Claim 1 we have

$$
w\left(\Gamma \cap D_{1}\right)=w\left(\Gamma \cap \partial D_{1}\right)+w\left(\Gamma \cap \operatorname{Int} D_{1}\right) \geq 3+2=5 .
$$

Since $D_{2}$ is a 2-angled disk, we have

$$
w\left(\Gamma \cap D_{2}\right)=w\left(\Gamma \cap \partial D_{2}\right)+w\left(\Gamma \cap \operatorname{Int} D_{2}\right)=2+w\left(\Gamma \cap \operatorname{Int} D_{2}\right) .
$$

Hence

$$
\begin{aligned}
7=w(\Gamma) & =w\left(\Gamma \cap D_{1}\right)+w\left(\Gamma \cap D_{2}\right)+w\left(\Gamma \cap\left(S^{2}-\left(D_{1} \cup D_{2}\right)\right)\right) \\
& \geq 5+2+w\left(\Gamma \cap \operatorname{Int} D_{2}\right)+w\left(\Gamma \cap\left(S^{2}-\left(D_{1} \cup D_{2}\right)\right)\right) .
\end{aligned}
$$

Thus $0 \geq w\left(\Gamma \cap \operatorname{Int} D_{2}\right)+w\left(\Gamma \cap\left(S^{2}-\left(D_{1} \cup D_{2}\right)\right)\right)$. Therefore Claim 2 holds.

Since $w\left(\Gamma \cap \operatorname{Int} D_{2}\right)=0$ by Claim 2, a neighborhood of $D_{2}$ contains the pseudo chart as shown in Fig. 10(a) by Lemma 5.2. Let $e_{1}$ be the terminal edge of label $m+1$ at $w_{1}$. Moreover, we have the following claim:

Claim 3. $e_{1} \not \subset D_{2}$ (see Fig. 23).

Let $a_{11}, b_{11}$ be internal edges (possibly terminal edges) of label $m+2$ such that $a_{11}, e_{1}, b_{11}$ are oriented inward at $w_{1}$ and lie anticlockwise around $w_{1}$. Then by Assumption 2 we have

(3) neither $a_{11}$ nor $b_{11}$ is a terminal edge.

Let $e_{5}$ be the terminal edge of label $m+1$ at $w_{5}$.

Claim 4. $e_{5} \not \subset D_{1}$.

Proof of Claim 4. Suppose $e_{5} \subset D_{1}$. Then there exists only one edge $e$ of label $m+2$ at $w_{5}$ in $C l\left(S^{2}-\left(D_{1} \cup D_{2}\right)\right.$ ) (see Fig. 23(a)). Moreover

(4) the edge $e$ is oriented inward at $w_{5}$.

Since $a_{11}, b_{11}, a_{23}$ are oriented inward at $w_{1}, w_{1}, w_{3}$ respectively (see Fig. 23(a)), and since neither $a_{11}$ nor $b_{11}$ is a terminal edge by (3), we have $w\left(\Gamma \cap\left(S^{2}-\right.\right.$ $\left.\left.\left(D_{1} \cup D_{2}\right)\right)\right) \geq 1$ by IO-Calculation with respect to $\Gamma_{m+2}$ in $C l\left(S^{2}-\left(D_{1} \cup D_{2}\right)\right)$. This contradicts Claim 2. Hence $e_{5} \not \subset D_{1}$. Thus Claim 4 holds.

Let $a_{55}, b_{55}$ be internal edges (possibly terminal edges) of label $m+2$ at $w_{5}$ such that $a_{55}, e_{5}, b_{55}$ are consecutive three edges lying anticlockwise around $w_{5}$. Then by Assumption 2 ,

(5) neither $a_{55}$ nor $b_{55}$ is a terminal edge.

Now we are ready to prove Proposition 10.3. By Claim 3 and Claim 4, the chart $\Gamma$ contains the pseudo chart as shown in Fig. 23(b). Since $b_{23}, a_{55}, b_{55}$ are oriented outward at $w_{3}, w_{5}, w_{5}$ respectively (see Fig. $23(\mathrm{~b})$ ), and since 
none of $b_{23}, a_{55}, b_{55}$ are terminal edges by (2) and (5), the condition $w(\Gamma \cap$ $\left.\left(S^{2}-\left(D_{1} \cup D_{2}\right)\right)\right)=0$ implies $b_{23}=b_{11}, a_{55}=a_{11}$ and $b_{55}=a_{23}$. Thus the simple closed curve $C=b_{23} \cup a_{55} \cup b_{55}$ of label $m+2$ contains only three white vertices $w_{1}, w_{3}, w_{5}$. Hence by Corollary 8.6, the graph $\Gamma_{m+2}$ contains the graph as shown in Fig. 5(a). Thus by Lemma 4.4, the simple closed curve $C$ contains one white vertex of $\Gamma_{m+3}$ (see Fig. 9(a),(b)). However all of the white vertices $w_{1}, w_{3}, w_{5}$ in $C$ are contained in $\Gamma_{m+1} \cap \Gamma_{m+2}$. This is a contradiction. Therefore $\Gamma_{m+1}$ does not contain the graph as shown in Fig. 5(a).

By Lemma 8.4, we can show that $\Gamma_{m+2}$ does not contain the graph as shown in Fig. 5(a). Therefore we complete the proof of Proposition 10.3 .
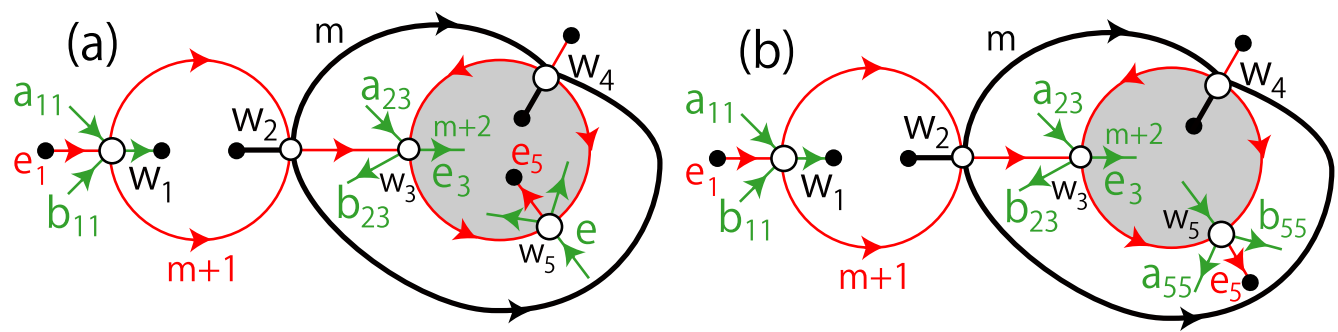

Figure 23: The gray regions are the disk $D_{1}$. (a) $e_{5} \subset D_{1}$. (b) $e_{5} \not \subset D_{1}$.

\section{Shifting Lemma}

In this section we give an example of a non minimal chart $\Gamma$ of type $(m ; 2,3,2)$ such that $\Gamma_{m+1}$ contains the graph as shown in Fig. 5(b).

Let $\Gamma$ be a chart. Let $\alpha$ be an arc in an edge of $\Gamma_{m}$, and $w$ a white vertex with $w \notin \alpha$. Suppose that there exists an $\operatorname{arc} \beta$ in $\Gamma$ such that its end points are the white vertex $w$ and an interior point $p$ of the $\operatorname{arc} \alpha$. Then we say that the white vertex $w$ connects with the point $p$ of $\alpha$ by the arc $\beta$.

Let $\alpha$ be a simple arc, and $p, q$ points in $\alpha$. We denote by $\alpha[p, q]$ the subarc of $\alpha$ whose endpoints are $p$ and $q$.

Lemma 11.1 ([6, Lemma 4.2]) (Shifting Lemma) Let $\Gamma$ be a chart and $\alpha$ an arc in an edge of $\Gamma_{m}$. Let $w$ be a white vertex of $\Gamma_{k} \cap \Gamma_{h}$ where $h=k+\varepsilon, \varepsilon \in$ $\{+1,-1\}$. Suppose that the white vertex $w$ connects with a point $r$ of the arc $\alpha$ by an arc in an edge e of $\Gamma_{k}$. Suppose that one of the following two conditions is satisfied:

(1) $h>k>m$.

(2) $h<k<m$. 
Then for any neighborhood $V$ of the arc $e[w, r]$ we can shift the white vertex $w$ to $e-e[w, r]$ along the edge e by $C$-I-R2 moves, $C$-I-R3 moves and C-I-R4 moves in $V$ keeping $\bigcup_{i<0} \Gamma_{k+i \varepsilon}$ fixed (see Fig.24).
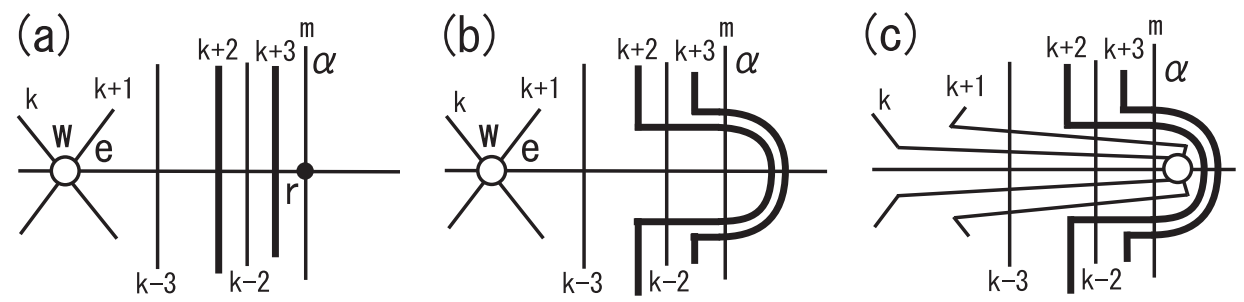

Figure 24: $\quad k>m$ and $\varepsilon=+1$.

Lemma 11.2 Let $\Gamma$ be a chart of type $(m ; 2,3,2)$ containing the pseudo chart as shown in Fig. $8(d)$. Let $e_{2}^{\prime}, e_{2}^{\prime \prime}, e_{3}^{\prime}, e_{3}^{\prime \prime}, e_{5}^{\prime}, e_{5}^{\prime \prime}$ be internal edges of label $m+2$ such that $e_{2}^{\prime}, e_{2}^{\prime \prime}$ are oriented outward at the white vertex $w_{2}, e_{3}^{\prime}, e_{3}^{\prime \prime}$ are oriented outward at the white vertex $w_{3}$, and $e_{5}^{\prime}, e_{5}^{\prime \prime}$ are oriented inward at the white vertex $w_{5}$. If $e_{2}^{\prime} \ni w_{3}, e_{3}^{\prime} \cap e_{3}^{\prime \prime}$ contains a white vertex different from $w_{3}$, and $e_{2}^{\prime \prime} \cap e_{5}^{\prime} \cap e_{5}^{\prime \prime}$ contains a white vertex (see Fig. 25(a)), then $\Gamma$ is not a minimal chart.

Proof. Let $w_{6}, w_{7}$ be the white vertices different from $w_{2}, w_{3}, w_{5}$ with $w_{6} \in$ $e_{3}^{\prime} \cap e_{3}^{\prime \prime}$ and $w_{7} \in e_{2}^{\prime \prime} \cap e_{5}^{\prime} \cap e_{5}^{\prime \prime}$. Let $\widetilde{e}$ be the internal edge of label $m+1$ with $\widetilde{e} \ni w_{2}, w_{3}$. Let $e_{3}$ be the terminal edge of label $m+1$ at $w_{3}$. Let $E$ be the disk with $\partial E=e_{2}^{\prime} \cup \widetilde{e}$ and $e_{3} \cap \operatorname{Int} E=\emptyset$. Then

(1) none of the five white vertices $w_{1}, w_{2}, \cdots, w_{5}$ are contained in $\operatorname{Int} E$,

(2) $\left(e_{2}^{\prime \prime} \cup e_{3}^{\prime}\right) \cap \operatorname{Int} E=\emptyset$.

Claim 1. $w(\Gamma \cap \operatorname{Int} E)=0$.

Proof of Claim 1. By (1), it suffices to prove that neither $w_{6}$ nor $w_{7}$ is contained in $\operatorname{Int} E$. Since the white vertices $w_{6}$ and $w_{7}$ are contained in the edges $e_{3}^{\prime}$ and $e_{2}^{\prime \prime}$ respectively, we have $w_{6} \notin \operatorname{Int} E$ and $w_{7} \notin \operatorname{Int} E$ by (2). Thus Claim 1 holds.

By Claim 1, we can apply New Disk Lemma (Lemma 8.1) for the disk $E$ so that we have the following claim:

Claim 2. We can assume that $\Gamma$ is $\left(E, e_{2}^{\prime}\right)$-arc free by C-moves in a neighborhood of $e_{2}^{\prime}$ keeping $\Gamma_{m+1} \cup \Gamma_{m+2}$ fixed.

Let $G, G_{m+1}$ be the connected components of $\Gamma_{m}, \Gamma_{m+1}$ respectively such that $w(G)=2$ and $w\left(G_{m+1}\right)=5$. Let $F$ be the closure of the connected component of $S^{2}-\left(G \cup G_{m+1}\right)$ with $\partial F \ni w_{1}, w_{2}, w_{3}, w_{4}$ and $e_{3} \cap \operatorname{Int} F=\emptyset$. Then 

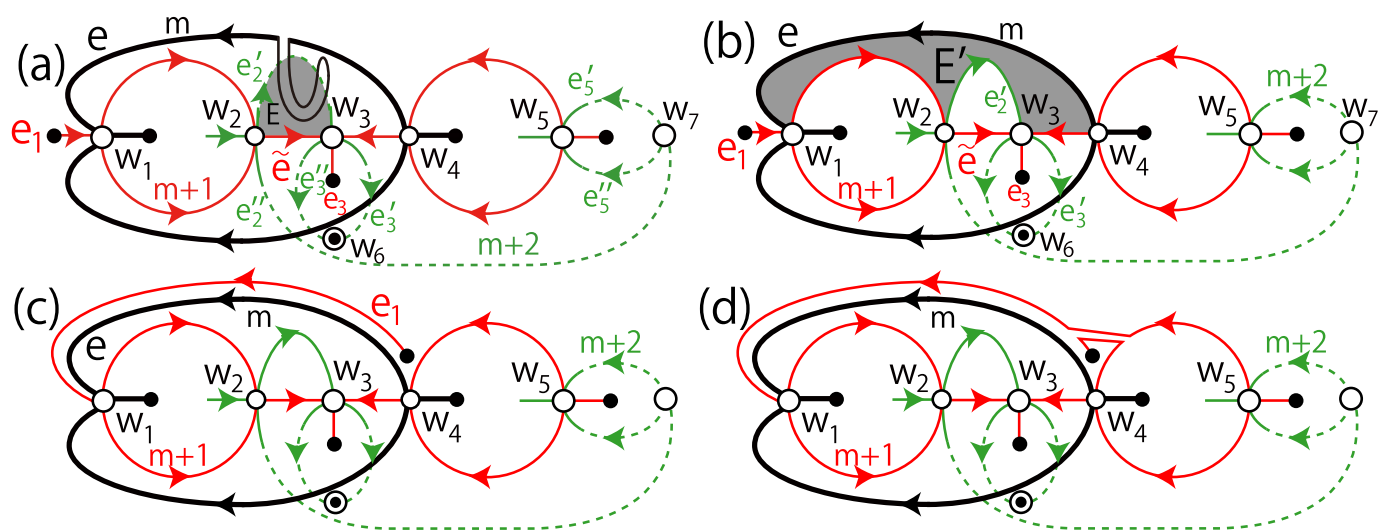

Figure 25: (a) The gray region is the disk E. (b) The gray region is the disk $E^{\prime}$. (c), (d) We apply C-II moves and a C-I-M2 move for the terminal edge $e_{1}$.

(3) none of the five white vertices $w_{1}, w_{2}, \cdots, w_{5}$ are contained in $\operatorname{Int} F$.

Let $e=\Gamma_{m} \cap \partial F$. Then $e$ is an internal edge of label $m$ connecting $w_{1}$ and $w_{4}$ (see Fig. 25(a)).

Claim 3. $e \cap e_{2}^{\prime}=\emptyset$ (see Fig. 25(b)).

Proof of Claim 3. Suppose $e \cap e_{2}^{\prime} \neq \emptyset$. By (1), the two white vertices $w_{1}$ and $w_{4}$ are in the outside of $E$. Thus the intersection $e \cap E$ consists of proper arcs of $E$. Since $\partial E=e_{2}^{\prime} \cup \widetilde{e}$ and since $\widetilde{e}$ is an edge of label $m+1$, the intersection $e \cap E$ consists of $\left(E, e_{2}^{\prime}\right)$-arcs of label $m$. This contradicts Claim 2. Thus $e \cap e_{2}^{\prime}=\emptyset$. Hence Claim 3 holds.

By Claim 3, the edge $e_{2}^{\prime}$ is a proper arc of $F$. Thus the edge $e_{2}^{\prime}$ separates the region $F$ into the disk $E$ and a disk, say $E^{\prime}$. Moreover

(4) the boundary $\partial E^{\prime}$ consists of $e, e_{2}^{\prime}$ and two internal edges of label $m+1$.

Since the five white vertices $w_{1}, w_{2}, \cdots, w_{5}$ are contained in $\Gamma_{m+1}$ and since $\Gamma$ is of type $(m ; 2,3,2)$, we have

(5) $w_{6}, w_{7} \in \Gamma_{m+2} \cap \Gamma_{m+3}$.

Claim 4. We can assume $w\left(\Gamma \cap \operatorname{Int} E^{\prime}\right)=0$ by C-moves in a neighborhood of $E^{\prime}$ keeping $\partial E^{\prime}$ fixed.

Proof of Claim 4. By (3), none of $w_{1}, w_{2}, \cdots, w_{5}$ are contained in $\operatorname{Int} E^{\prime}$. Thus it is possible that Int $E^{\prime}$ contains $w_{6}$ or $w_{7}$.

Suppose that $\operatorname{Int} E^{\prime}$ contains the white vertex $w_{6}$. Since the edge $e_{3}^{\prime}$ of label $m+2$ connects $w_{6}$ and $w_{3}$, the edge $e_{3}^{\prime}$ intersects $\partial E^{\prime}-w_{3}$. Hence by (4), the edge $e_{3}^{\prime}$ intersects the edge $e$ of label $m$. Let $x$ be the point in $e_{3}^{\prime}$ with $e_{3}^{\prime}\left[w_{6}, x\right] \cap e=x$. Then by (5) the vertex $w_{6} \in \Gamma_{m+2} \cap \Gamma_{m+3}$ connects the point $x$ of the edge $e$ of label $m$ by the arc $e_{3}^{\prime}\left[w_{6}, x\right]$ of label $m+2$. Hence by Shifting Lemma (Lemma 11.1), we can shift the white vertex $w_{6}$ to the 
outside of $E^{\prime}$ along the arc $e_{3}^{\prime}\left[w_{6}, x\right]$ by C-I-R2 moves, C-I-R3 moves and C-I-R4 moves in a neighborhood of the arc $e_{3}^{\prime}\left[w_{6}, x\right]$ keeping $\bigcup_{i<0} \Gamma_{m+2+i}$ fixed. Thus we can shift the white vertex $w_{6}$ to the outside of $E^{\prime}$ keeping $\partial E^{\prime}$ fixed.

Similarly we can shift the white vertex $w_{7}$ to the outside of $E^{\prime}$ keeping $\partial E^{\prime}$ fixed. Hence Claim 4 holds.

By Claim 4, we can apply New Disk Lemma (Lemma 8.1) for the disk $E^{\prime}$ so that we have the following claim:

Claim 5. We can assume that $\Gamma$ is $\left(E^{\prime}, e\right)$-arc free by $\mathrm{C}$-moves in a neighborhood of the edge $e$ keeping $\partial E^{\prime}$ fixed.

By the similar way of the proof of Claim 3, we have the following claim:

Claim 6. $\Gamma_{m+2} \cap e=\emptyset$.

Now we are ready to prove Lemma 11.2. Suppose that $\Gamma$ is minimal. By Claim 6, we can apply C-II moves along the edge $e$ for the black vertex in the terminal edge $e_{1}$ of label $m+1$ at $w_{1}$ such that the black vertex in $e_{1}$ is near the white vertex $w_{4}$ (see Fig. 25(c)). By a C-I-M2 move between $e_{1}$ and an internal edge of label $m+1$ connecting $w_{4}$ and $w_{5}$, we obtain a new terminal edge of label $m+1$ at $w_{4}$ but not middle at $w_{4}$ (see Fig. 25(d)). This contradicts Assumption 2. Therefore $\Gamma$ is not minimal. We complete the proof of Lemma 11.2 .

\section{Proof of Main Theorem}

In this section, we shall show the main theorem (Theorem 1.1).

Lemma 12.1 Let $\Gamma$ be a chart of type $(m ; 2,3,2)$. If $\Gamma$ contains the pseudo chart as shown in Fig. $8(c)$, then $\Gamma$ is not minimal.

Proof. Suppose that $\Gamma$ is minimal. We use the notations as shown in Fig. 8(c) where

(1) $e_{2}, e_{4}$ are internal edges (possibly terminal edges) of label $m+2$ middle at $w_{2}, w_{4}$ and oriented inward at $w_{2}, w_{4}$ respectively.

Since $w_{1}, w_{3} \in \Gamma_{m} \cap \Gamma_{m+1}, w_{2}, w_{4}, w_{5} \in \Gamma_{m+1}$ and $\Gamma$ is of type $(m ; 2,3,2)$, we have

(2) $\Gamma_{m+1} \cap \Gamma_{m+2}=\left\{w_{2}, w_{4}, w_{5}\right\}$.

By Corollary 8.6 and Proposition 10.3 , the graph $\Gamma_{m+2}$ contains the graph as shown in Fig. 5(b). Hence by using Lemma 3.1, the graph $\Gamma_{m+2}$ contains one of the two graphs as shown in Fig. 26(a),(b). We also use the notations as shown in Fig. 26(a),(b) where $v_{1}, v_{2}, \cdots, v_{5}$ are the white vertices in $\Gamma_{m+2}$, and 
(3) $\widetilde{e}_{2}, \widetilde{e}_{4}$ are internal edges of label $m+2$ middle at $v_{2}, v_{4}$ respectively,

(4) the edge of label $m+2$ middle at $v_{5}$ is a terminal edge.

Claim. $w_{2} \neq v_{2}$ and $w_{2} \neq v_{4}$.

Proof of Claim. If $w_{2}=v_{2}$, then $e_{2}$ is middle at $w_{2}\left(=v_{2}\right)$ by (1). Thus by (3) we have $e_{2}=\widetilde{e}_{2}$.

Let $D$ be the 2-angled disk of $\Gamma_{m+1}$ with $\partial D \ni w_{1}, w_{2}$ and $D \supset e_{2}\left(=\widetilde{e}_{2}\right)$ (see Fig. 8(c) and Fig. 26(c)). Since $w_{1} \in \Gamma_{m} \cap \partial D$, the interior $\operatorname{Int} D$ contains $v_{3}, v_{4}$ and $v_{5}$ (see Fig. $\left.26(\mathrm{c})\right)$. Thus $w(\Gamma \cap \operatorname{Int} D) \geq 3$. Since $\Gamma$ is of type $(m ; 2,3,2)$, we have $w\left(\overline{\Gamma)}=7\right.$ and $w\left(\Gamma_{m+1}\right)=5$. Hence

$$
7=w(\Gamma) \geq w\left(\Gamma_{m+1}\right)+w(\Gamma \cap \operatorname{Int} D) \geq 5+3=8 .
$$

This is a contradiction. Thus $w_{2} \neq v_{2}$.

Similarly we can show that $w_{2} \neq v_{4}$. Hence Claim holds.

Since $\Gamma_{m+2}$ contains the graph as shown in Fig. 5(b), by Lemma 4.4 the chart $\Gamma$ contains one of the RO-families of the two pseudo charts as shown in Fig. 9(c),(d). Hence there are two cases: (i) $v_{1}, v_{3} \in \Gamma_{m+3}$ or $v_{3}, v_{5} \in \Gamma_{m+3}$, (ii) $v_{1}, v_{4} \in \Gamma_{m+3}$ or $v_{2}, v_{5} \in \Gamma_{m+3}$.

Case (i). Without loss of generality we can assume $v_{1}, v_{3} \in \Gamma_{m+3}$. Since $v_{1}, v_{2}, v_{3}, v_{4}, v_{5} \in \Gamma_{m+2}$ and $\Gamma$ is of type $(m ; 2,3,2)$, we have $\Gamma_{m+1} \cap \Gamma_{m+2}=$ $\left\{v_{2}, v_{4}, v_{5}\right\}$. Thus by $(2)$ we have $\left\{v_{2}, v_{4}, v_{5}\right\}=\left\{w_{2}, w_{4}, w_{5}\right\}$. Hence by Claim, we have $w_{2}=v_{5}$ and

(5) $w_{4} \in\left\{v_{2}, v_{4}\right\}$.

Since the edge $e_{2}$ of label $m+2$ is middle at $w_{2}\left(=v_{5}\right)$ by $(1)$, the edge $e_{2}$ is the terminal edge at $v_{5}$ by (4). Moreover by (1), the terminal edge $e_{2}$ is oriented inward at $w_{2}\left(=v_{5}\right)$. Thus $\Gamma_{m+2}$ contains the graph as shown in Fig. 26(a). Hence

(6) the two edges $\widetilde{e}_{2}, \widetilde{e}_{4}$ of label $m+2$ are oriented outward at $v_{2}, v_{4}$ respectively.

On the other hand, since the edge $e_{4}$ of label $m+2$ is middle at $w_{4}$ by (1), either $e_{4}=\widetilde{e}_{2}$ or $e_{4}=\widetilde{e}_{4}$ by (3) and (5). Since the edge $e_{4}$ of label $m+2$ is oriented inward at $w_{4}$ by (1), one of the two edges $\widetilde{e}_{2}, \widetilde{e}_{4}$ is oriented inward at $w_{4}\left(v_{2}\right.$ or $\left.v_{4}\right)$. This contradicts (6). Hence Case (i) does not occur.

Case (ii). Without loss of generality we can assume $v_{1}, v_{4} \in \Gamma_{m+3}$. Since $v_{1}, v_{2}, v_{3}, v_{4}, v_{5} \in \Gamma_{m+2}$ and $\Gamma$ is of type $(m ; 2,3,2)$, we have $\Gamma_{m+1} \cap \Gamma_{m+2}=$ $\left\{v_{2}, v_{3}, v_{5}\right\}$. Thus by (2) we have

(7) $\left\{v_{2}, v_{3}, v_{5}\right\}=\left\{w_{2}, w_{4}, w_{5}\right\}$.

Let $e_{5}$ be the internal edge (possibly terminal edge) of label $m+2$ middle at $w_{5}$, and $\widetilde{e}_{3}, \widetilde{e}_{5}$ the terminal edges of label $m+2$ at $v_{3}, v_{5}$ respectively. Since $\widetilde{e}_{3}, \widetilde{e}_{5}$ are middle at $v_{3}, v_{5}$ respectively by Assumption 2 and since $e_{2}, e_{4}, \widetilde{e}_{2}$ are middle at $w_{2}, w_{4}, v_{2}$ respectively by (1) and (3), the condition (7) implies that 
(8) $\left\{\widetilde{e}_{2}, \widetilde{e}_{3}, \widetilde{e}_{5}\right\}=\left\{e_{2}, e_{4}, e_{5}\right\}$.

Since there are two internal edges of label $m+1$ oriented from $w_{5}$ to $w_{4}$ (see Fig. $8(\mathrm{c})$ ), the edge $e_{5}$ is oriented outward at $w_{5}$. Moreover since $e_{2}, e_{4}$ are oriented inward at $w_{2}, w_{4}$ respectively by (1), the condition (8) implies that the graph $\Gamma_{m+2}$ contains the graph as shown in Fig. 26(b) and $\left\{w_{2}, w_{4}\right\}=\left\{v_{2}, v_{3}\right\}, w_{5}=v_{5}$. Thus by Claim, we have $w_{2}=v_{3}$ and $w_{4}=v_{2}$. Hence the condition $w_{4}=v_{2}$ implies $e_{4}=\widetilde{e}_{2}$. Thus the edge $e_{4}\left(=\widetilde{e}_{2}\right)$ connects $w_{4}\left(=v_{2}\right)$ and $w_{2}\left(=v_{3}\right)$ (see Fig. 26(b)). However the edge $e_{4}$ must intersect the boundary of the 2-angled disk of $\Gamma_{m+1}$ (see Fig. 26(d)). This contradicts the definition of a crossing. Thus Case (ii) does not occur.

Therefore $\Gamma$ is not minimal. We complete the proof of Lemma 12.1.
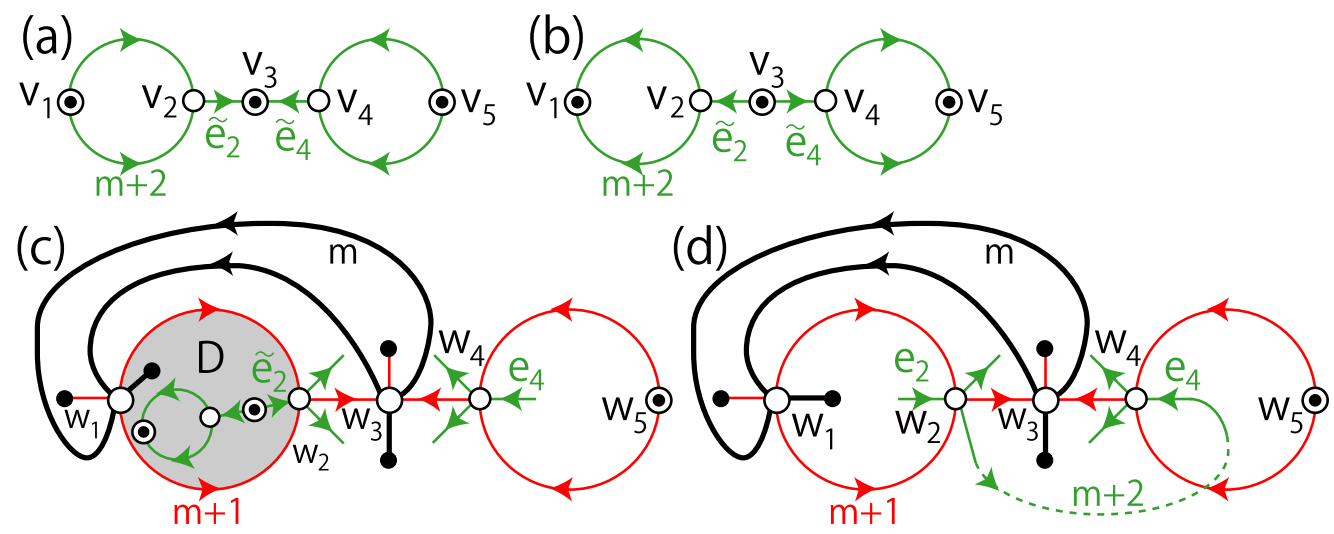

Figure 26: (a), (b) The graphs as shown in Fig. 5(b). (c) The disk $D$ contains at least three white vertices in its interior. (d) There is an internal edge of label $m+2$ connecting $w_{2}$ and $w_{4}$.

Proof of Theorem 1.1. Suppose that there exists a minimal chart $\Gamma$ of type $(m ; 2,3,2)$. By Corollary 8.6 and Proposition 10.3 , each of $\Gamma_{m+1}$ and $\Gamma_{m+2}$ contains the graph as shown in Fig. 5(b). By Lemma 4.3 and Lemma 12.1. the chart $\Gamma$ contains one of the RO-family of the pseudo chart as shown in Fig. 8(d). We use the notations as shown in Fig. 8(d) where

(1) $e_{2}$ is an internal edge (possibly terminal edge) of label $m+2$ middle at $w_{2}$ and oriented inward at $w_{2}$.

Since $w_{1}, w_{4} \in \Gamma_{m} \cap \Gamma_{m+1}, w_{2}, w_{3}, w_{5} \in \Gamma_{m+1}$ and $\Gamma$ is of type $(m ; 2,3,2)$, we have

(2) $\Gamma_{m+1} \cap \Gamma_{m+2}=\left\{w_{2}, w_{3}, w_{5}\right\}$

Since $\Gamma_{m+2}$ contains the graph as shown in Fig. 5 (b), the graph $\Gamma_{m+2}$ contains one of the two graphs as shown in Fig. 26(a),(b). We also use the notations as shown in Fig. 26(a),(b), where $v_{1}, v_{2}, \cdots, v_{5}$ are the white vertices in $\Gamma_{m+2}$, 
(3) $\widetilde{e}_{2}$ is middle at $v_{2}$.

Moreover, by Lemma 4.4 , the chart $\Gamma$ contains one of the RO-families of the two pseudo charts as shown in Fig. 9(c),(d). Hence there are two cases: (i) $v_{1}, v_{3} \in \Gamma_{m+3}$ or $v_{3}, v_{5} \in \Gamma_{m+3}$, (ii) $v_{1}, v_{4} \in \Gamma_{m+3}$ or $v_{2}, v_{5} \in \Gamma_{m+3}$.

Case (i). By changing the labels $\cdots, m, m+1, m+2, m+3, \cdots$ into $\cdots, m+3, m+2, m+1, m, \cdots$ respectively, the chart $\Gamma$ contains the pseudo chart as shown in Fig. 8(c). Thus by Lemma 12.1, we show that $\Gamma$ is not a minimal chart. This is a contradiction. Hence Case (i) does not occur.

Case (ii). Without loss of generality we can assume $v_{1}, v_{4} \in \Gamma_{m+3}$. Since $v_{1}, v_{2}, v_{3}, v_{4}, v_{5} \in \Gamma_{m+2}$ and $\Gamma$ is of type $(m ; 2,3,2)$, we have $\Gamma_{m+1} \cap \Gamma_{m+2}=$ $\left\{v_{2}, v_{3}, v_{5}\right\}$. Thus by (2), we have

(4) $\left\{v_{2}, v_{3}, v_{5}\right\}=\left\{w_{2}, w_{3}, w_{5}\right\}$.

By the same way of the proof of Claim in Lemma 12.1, we can show that

(5) $w_{2} \neq v_{2}$ and $w_{2} \neq v_{4}$.

Let $e_{3}, e_{5}$ be internal edges (possibly terminal edges) of label $m+2$ middle at $w_{3}, w_{5}$ respectively. Let $\widetilde{e}_{3}, \widetilde{e}_{5}$ be the terminal edges at $v_{3}, v_{5}$ respectively. Since $\widetilde{e}_{3}, \widetilde{e}_{5}$ are middle at $v_{3}, v_{5}$ respectively by Assumption 2 and since $e_{2}, \widetilde{e}_{2}$ are middle at $w_{2}, v_{2}$ respectively by (1) and (3), the condition (4) implies that

(6) $\left\{\widetilde{e}_{2}, \widetilde{e}_{3}, \widetilde{e}_{5}\right\}=\left\{e_{2}, e_{3}, e_{5}\right\}$.

Since there are two internal edges of label $m+1$ oriented inward at $w_{3}$, and since there are two internal edges of label $m+1$ oriented outward at $w_{5}$ (see Fig. $8(\mathrm{~d})$ ),

(7) the edge $e_{3}$ is oriented inward at $w_{3}$ and the edge $e_{5}$ is oriented outward at $w_{5}$.

Furthermore since $e_{2}$ is oriented inward at $w_{2}$ by (1), the conditions (6) and (7) imply that $\Gamma_{m+2}$ contains the graph as shown in Fig. 26(b). Hence $\left\{w_{2}, w_{3}\right\}=\left\{v_{2}, v_{3}\right\}, w_{5}=v_{5}$. Thus by (5), we have $w_{2}=v_{3}$ and $w_{3}=v_{2}$. Hence $e_{2}=\widetilde{e}_{3}, e_{3}=\widetilde{e}_{2}$ and $e_{5}=\widetilde{e}_{5}$. Hence $e_{3}\left(=\widetilde{e}_{2}\right) \ni w_{2}\left(=v_{3}\right), w_{3}\left(=v_{2}\right)$, and both of $e_{2}\left(=\widetilde{e}_{3}\right), e_{5}\left(=\widetilde{e}_{5}\right)$ are terminal edges. Furthermore, there exist two internal edges of label $m+2$ connecting $w_{3}\left(=v_{2}\right)$ and $v_{1}$, and there exist three internal edges of label $m+2$ at $v_{4}$ containing $w_{2}\left(=v_{3}\right), w_{5}\left(=v_{5}\right), w_{5}$ respectively. Therefore $\Gamma$ satisfies the condition of Lemma 11.2 (see Fig. 25(a)). By Lemma 11.2, the chart $\Gamma$ is not minimal. This is a contradiction. Hence Case (ii) does not occur.

Therefore both cases do not occur. Hence there does not exist any minimal chart of type $(m ; 2,3,2)$. We complete the proof of Theorem 1.1 . 


\section{References}

[1] J. S. Carter and M. Saito, "Knotted surfaces and their diagrams", Mathematical Surveys and Monographs, 55, American Mathematical Society, Providence, RI, (1998). MR1487374 (98m:57027)

[2] I. Hasegawa, The lower bound of the w-indices of non-ribbon surfacelinks, Osaka J. Math. 41 (2004), 891-909. MR2116344 (2005k:57045)

[3] S. Ishida, T. Nagase and A. Shima, Minimal n-charts with four white vertices, J. Knot Theory Ramifications 20, 689-711 (2011). MR2806339 (2012e:57044)

[4] S. Kamada, Surfaces in $R^{4}$ of braid index three are ribbon, J. Knot Theory Ramifications 1 No. 2 (1992), 137-160. MR1164113 (93h:57039)

[5] S. Kamada, "Braid and Knot Theory in Dimension Four", Mathematical Surveys and Monographs, Vol. 95, American Mathematical Society, (2002). MR1900979 (2003d:57050)

[6] T. Nagase and A. Shima, Properties of minimal charts and their applications I, J. Math. Sci. Univ. Tokyo 14 (2007), 69-97. MR2320385 (2008c:57040)

[7] T. Nagase and A. Shima, Properties of minimal charts and their applications II, Hiroshima Math. J. 39 (2009), 1-35. MR2499196 (2009k:57040)

[8] T. Nagase and A. Shima, Properties of minimal charts and their applications III, Tokyo J. Math. 33 (2010), 373-392. MR2779264 (2012a:57033)

[9] T. Nagase and A. Shima, Properties of minimal charts and their applications IV: Loops, J. Math. Sci. Univ. Tokyo 24 (2017), 195-237, arXiv:1603.04639, MR3674447

[10] T. Nagase and A. Shima, Properties of minimal charts and their applications $V$ : charts of type $(3,2,2)$, J. Knot Theory Ramifications 28 No. 14 (2019) 1950084 (27 pages), arXiv:1902.00007.

[11] T. Nagase and A. Shima, Properties of minimal charts and their applications VI: the graph $\Gamma_{m+1}$ in a chart $\Gamma$ of type $(m ; 2,3,2)$, preprint, arXiv:2003.11909.

[12] T. Nagase and A. Shima, Properties of minimal charts and their applications VIII-, in preparation.

[13] T. Nagase and A. Shima, Minimal charts of type (3,3), Proc. Sch. Sci. TOKAI UNIV. 52 (2017), 1-25, arXiv:1609.08257v2. 
[14] T. Nagase and A. Shima, The structure of a minimal n-chart with two crossings I: Complementary domains of $\Gamma_{1} \cup \Gamma_{n-1}$, J. Knot Theory Ramifications 27 No. 14 (2018) 1850078 (37 pages), arXiv:1704.01232v3.

[15] T. Nagase, A. Shima and H. Tsuji, The closures of surface braids obtained from minimal n-charts with four white vertices, J. Knot Theory Ramifications 22 No. 2 (2013) 1350007 (27 pages). MR3037298

[16] M. Ochiai, T. Nagase and A. Shima, There exists no minimal n-chart with five white vertices, Proc. Sch. Sci. TOKAI UNIV. 40 (2005), 1-18. MR2138333 (2006b:57035)

[17] K. Tanaka, A Note on CI-moves, Intelligence of low dimensional topology 2006, 307-314, Ser. Knots Everything, 40, World Sci. Publ., Hackensack, NJ, 2007. MR2371740 (2009a:57017)

Teruo NAGASE

Tokai University

4-1-1 Kitakaname, Hiratuka

Kanagawa, 259-1292 Japan

nagase@keyaki.cc.u-tokai.ac.jp
Akiko SHIMA

Department of Mathematics,

Tokai University

4-1-1 Kitakaname, Hiratuka

Kanagawa, 259-1292 Japan

shima@keyaki.cc.u-tokai.ac.jp

\section{List of terminologies}

\begin{tabular}{|c|c|c|c|}
\hline$k$-angled disk & $p 8$ & middle arc & $p 3$ \\
\hline BW-vertex & $p 5$ & middle at $v$ & $p 3$ \\
\hline C-move equivalent & $p 3$ & minimal chart & $p 3$ \\
\hline hart & $p 2$ & outward & $p 3$ \\
\hline complexity & $p 3$ & outward arc & $p 1$ \\
\hline$(D, \alpha)$-arc of label $k$ & $p 16$ & oval & $p 5$ \\
\hline$(D, \alpha)$-arc free & $p 16$ & point at infinity $\infty$ & $p 4$ \\
\hline feeler & $p 8$ & proper arc & $p 9$ \\
\hline free edge & $p 3$ & pseudo chart & $p 6$ \\
\hline hoop & $p 4$ & ring & $p 4$ \\
\hline internal edge & $p 9$ & RO-family & $p 6$ \\
\hline inward & $p 3$ & simple hoop & $p 4$ \\
\hline inward arc & $p 10$ & skew $\theta$-curve & $p 5$ \\
\hline IO-Calculation & $p 11$ & special $k$-angled disk & $p$ \\
\hline keeping $X$ fixed & $p 12$ & special oval & $p$ \\
\hline local complexity & $p 21$ & terminal edge & $p$ \\
\hline locally minimal & $p 21$ & type $\left(m ; n_{1}, n_{2}, \cdots, n_{k}\right)$ & $P$ \\
\hline loop & $p 23$ & $w$ connects with $p$ by an $\operatorname{arc} \beta$ & \\
\hline
\end{tabular}


List of notations

$\begin{array}{ll}\Gamma_{m} & p 1 \\ \operatorname{Int} X & p 5 \\ \partial X & p 5 \\ C l(X) & p 5 \\ \partial \alpha & p 5 \\ \operatorname{Int} \alpha & p 5 \\ w(X) & p 5 \\ a_{i j}, b_{i j} & p 6 \\ c(X) & p 20 \\ \ell c(D ; \Gamma) & p 21 \\ \alpha[p, q] & p 28\end{array}$

